\title{
Neurotoxic astrocytes secreted glypican-4 drives Alzheimer's tau pathology
}

Ana Pereira ( $\square$ ana.pereira@mssm.edu )

Icahn School of Medicine at Mount Sinai https://orcid.org/0000-0003-2524-7969

Sivaprakasam Ramamoorthy

Icahn School of Medicine at Mount Sinai

Article

Keywords: Apolipoprotein E4, Alzheimer's disease, astrocyte-secreted protein glypican-4

Posted Date: October 1st, 2020

DOI: https://doi.org/10.21203/rs.3.rs-82205/v1

License: (c) (i) This work is licensed under a Creative Commons Attribution 4.0 International License. Read Full License 


\title{
Neurotoxic astrocytes secreted glypican-4 drives Alzheimer's tau pathology
}

\author{
Sivaprakasam R. Saroja ${ }^{1,2}$ and Ana C. Pereira ${ }^{1,2,3}$ \\ ${ }^{1}$ Department of Neurology, Icahn School of Medicine, Mount Sinai, New York, NY, 10029, \\ USA. \\ ${ }^{2}$ Nash Family Department of Neuroscience, Friedman Brain Institute, Icahn School of Medicine \\ at Mount Sinai, New York, NY, 10029, USA. \\ ${ }^{3}$ Ronald M. Loeb Center for Alzheimer's Disease, Icahn School of Medicine at Mount Sinai, \\ New York, NY, 10029, USA. \\ Corresponding author: Ana C. Pereira: ana.pereira@mssm.edu
}

\begin{abstract}
Apolipoprotein E4 (APOE4) is the most crucial genetic risk factor of late-onset Alzheimer's disease (AD). However, the mechanism through which APOE4 induces AD risk remains unknown. Here, we report the astrocyte-secreted protein glypican-4 (GPC-4), as a novel binding partner of APOE4, drives tau pathology. APOE4-carrying AD patients display more tau accumulation compared to APOE4-noncarring AD patients. GPC-4 is highly expressed in APOE4 AD patients, and is regulated by microglial factors via NF- $\kappa \mathrm{B}$ signaling pathway. The astrocyte-secreted GPC4 induced both tau accumulation and spreading in vitro and in vivo. Further, GPC-4 is required for APOE4-mediated surface trafficking of low-density lipoprotein receptor-related protein 1 (LRP1) and tau propagation. GPC-4 activates unfolded protein response (UPR) pathway IRE1 $\alpha$, and pharmacological inhibition of IRE1 $\alpha$ with KIRA6 blocks GPC-4 induced tau propagation. Together, our data comprehensively demonstrate that the APOE4-induced AD risk is directly mediated by GPC-4, and that perturbing GPC-4 induced IRE1 $\alpha$ pathway has therapeutic opportunities.
\end{abstract}

\section{INTRODUCTION}

Apolipoprotein (APOE) plays a major role in the circulation of high-density and very-low-density lipoproteins and mediates the transport of fats between the cells ${ }^{1}$. In the brain, APOE is secreted by glial cells, primary astrocytes ${ }^{2}$. Cholesterol released by astrocytes in the form of APOEcontaining high-density lipoprotein-like particles are vital for neuronal survival ${ }^{3}$. 
Human APOE is expressed in three genetic variants; APOE2, APOE3, and APOE4. These variants differ in terms of position of two amino acid residues; APOE3 has a cysteine at position 112 and arginine at position 158, APOE2 has a cysteine at both positions, and APOE4 has an arginine at both position ${ }^{4}$. Among the three APOE isoforms, APOE4 is the most crucial genetic risk factor for late-onset Alzheimer's disease (AD). The increase in AD risk varies depending on ancestral background, sex and multiple genetic or environmental factors, but as a rough estimate, having a single APOE4 allele increases AD risk 2- to 4-fold and having two APOE4 alleles increases AD risk about 8- to 12 -fold ${ }^{2}$. The APOE4 carriers also develop AD pathologies earlier compared to non-carriers ${ }^{2,5}$. By contrast, APOE2 carriers have a lower likelihood of developing AD; therefore, APOE2 protects against $\mathrm{AD}^{5-10}$.

$\mathrm{A} \beta$ plaques and neurofibrillary tau tangles are characteristic features of AD pathology ${ }^{11,12}$. AD patients with cognitive impairment often show a strong correlation with tau spreading ${ }^{13-18}$. A APOE3 Christchurch mutation (R136S) in a presenilin 1 mutation carrying individual (early-onset AD) accumulated less tau compared to control AD patients ${ }^{14}$. Though R136S carrying individual accumulated more brain amyloid levels, she did not develop any cognitive decline due to absence of tau accumulation. It suggests that abnormal tau plays a major role in cognitive impairments of AD patients. AD patients homozygous for APOE4 suffer from major cerebral atrophy ${ }^{19-21}$ and animal studies have demonstrated that pathological tau drives the cerebral atrophy ${ }^{22,23}$. However, it is not yet understood the mechanisms that drives tau pathology in AD patients and make APOE4 carriers more vulnerable to develop AD. Here, we comprehensively demonstrate that astrocyte secreted factor GPC-4 drives tau pathology.

\section{RESULTS}

\section{Human APOE4/4 Alzheimer's patients display more tau accumulation}

The abnormal posttranslational modification of tau protein is associated with formation of neurofibrillary tangles and prion-like propagation of tau proteins ${ }^{24,25}$. Both AD mouse models and human AD PET images suggest the abnormal tau as a major driver of cerebral atrophy, and APOE4 carrying AD patients display a severe atrophy compared to APOE4 noncarriers ${ }^{19,22}$. We therefore speculated that APOE4 carrying AD patients would have more tau accumulation. We performed immunohistochemical (IHC) staining with phosphorylated tau antibody AT8 (Ser202 and Thr205) 
and acetylated tau antibody (Lys174) on postmortem tissues of APOE2/3 (control), APOE2/2 (AD), APOE3/3 (AD) and APOE4/4(AD) individuals. There is no significant tau accumulation in APOE2/2 AD and APOE2/3 control (Fig. 1A). We observed presence of neurofibrillary tangles, neuropil threads and neuritic plaque both in APOE3/3 AD and APOE4/4 AD patients (Fig. 1A). The neurofibrillary tangles, neuropil threads and neuritic plaque are different forms of tauassociated neurofibrillary changes observed in the AD brain ${ }^{26}$. Interestingly, this triad of tau pathologies is significantly increased in APOE4/4 AD compared to APOE3/3 AD (Fig. 1B). Our data shows an accumulation of more tau protein in APOE4/4 AD patients compared to other APOE variants.

\section{APOE4 and APOE2 differentially affect tau propagation}

A majority of lipid-binding APOE proteins exist as oligomeric form in peripheral tissues ${ }^{27-29}$. Interestingly, immunoprecipitation from APOE44 human brains revealed that the majority of APOE4 protein exists as a monomeric protein or very small APOE4-containing particles (Fig. S1A), suggesting the presence of abundant lipid-free APOE4 proteins/very small particles in human brains. Further, tau seeding biosensor assay showed the APOE4-containing particles and purified human APOE4 protein induced tau seeding in similar ways (Fig. S1B and S1C). It is consistent with previous studies ${ }^{30,31}$; suggesting that APOE4 protein, not lipids, possess pathogenic activity.

By using a primary neuronal culture from the human tauopathy mouse model P301S, we first investigated which APOE variant is crucial for tau protein phosphorylation (Fig. S2A). We found that APOE2 significantly reduced tau phosphorylation compared to control and APOE4 treatments (Fig. S2C), while the total tau (tau-5) remained unchanged (Fig. S2B). Further, addition of both APOE2 and APOE4 also reduced AT8 levels (Fig. S2C). To address APOE2-mediated neuronal tau phosphorylation, as tau accumulation is also observed in glial cells ${ }^{32}$, we performed IHC in the presence of APOE2, APOE4, and both APOE2 and APOE4 (Fig. S2D). We observed the APOE2 significantly reduced AT8 levels in neurons (Fig. S2E).

We next determined whether APOE2 and APOE4 differentially influence tau spreading. For this purpose, we cocultured neurons from tau KO mice that express GFP while tau is absent and from P301S mice. With the cocultured neurons from these two mice, we expect that because the GFP+ 
neurons (tau KO) do not express endogenous tau proteins, the tau+ signal in GFP+ neurons can be considered to be the tau protein from P301S neurons. As expected, we observed the tau+ signal from the GFP+ neurons (tau KO neurons), suggesting the tau proteins are released by P301S neurons and were taken up by GFP+ neurons. (Fig. S2F). When the neuronal cultures were treated with APOE isoforms, we found that APOE4 robustly enhanced tau spreading (Fig. 1C, D). Upon adding both APOE2 and APOE4 to the neuronal cultures, we observed no additional increase in tau spreading because APOE2 hampers both tau phosphorylation and spread. It suggests that APOE2 induces a protective response by decreasing the AT8 levels and APOE4-induced tau spreading.

\section{Astrocyte secreted glypican-4 induces tau pathology}

How do the APOE variants differentially induce tau pathology? Alboleda-Velasquez et al recently reported that, compared to APOE2, APOE4 showed a strong interaction with heparin ${ }^{14}$. Therefore, we hypothesized that Heparan Sulfate Proteoglycans (HSPGs) may be involved in APOE4mediated tau pathology. To determine which HSPGs are crucial for tau pathology, we screened a list of HSPGs in vitro and found that glypican-4 (GPC-4) robustly induced AT8 levels in neuronal cultures from P301S animals (Fig. 2A-C). The IHC experiment yielded essentially the same result (Fig. 2D, E). To test the effect of GPC-4 on tau spreading, we cocultured neurons of P301S and tau KO animals and found that GPC-4 increased tau spreading from P301S neurons to tau KO neurons (Fig. 2F, G).

The GPC-4 is primarily expressed in astrocytes ${ }^{33}$. To validate whether astrocyte-secreted GPC-4 is sufficient to induce tau pathology, we treated P301S neuronal culture with astrocyte-conditioned medium (ACM) as described in Figure 2H. ACM-treated neurons expressed significantly higher levels of AT8 (Fig. 2I-K). We next treated the astrocytes with GPC-4 shRNA, and collected GPC4 deprived ACM (Figs. 2L). Interestingly, GPC-4 deprived ACM failed to induce tau pathology (Fig. 2M-O). It suggests that astrocyte-derived GPC-4 is sufficient to induce tau pathology.

\section{Neurotoxic astrocytes secrete glypican-4}

Given that APOE4 carrying AD patients displayed an accumulation of more tau proteins, we next investigated whether GPC-4 is differentially expressed in APOE variants. We found that APOE4- 
carrying $\mathrm{AD}$ patients expressed more GPC-4 protein compared to APOE4-noncarrying AD patients (Fig. 3A). Immunostaining from human postmortem brain tissues revealed that indeed astrocytes express GPC-4 protein (Fig. 3B). Single-cell RNA-sequencing (ScRNAseq) revealed the presence of $\mathrm{AD}$-associated genes and astrocytes in humans ${ }^{34-36}$. We therefore wondered which subtype of astrocytes express GPC-4. We generated a heatmap with those genes to investigate which subtype of astrocytes express GPC-4 (Fig. 3C). AD-genes are mainly expressed in astrocyte subtype 2 and 3 , suggesting that these are the clusters which are associated with AD. Interestingly, GPC-4 is expressed within AD associated astrocyte subcluster 3 (Fig. 3C).

A1 astrocytes which are induced by microglial factors TNF- $\alpha$ and IL-1 $\beta$ are considered as neurotoxic astrocytes ${ }^{37}$. As such, we hypothesized that microglial secretory factors-treated astrocytes would express GPC-4. As expected, TNF- $\alpha$ and IL-1 $\beta$-treated astrocytes expressed significantly higher levels of GPC-4 protein (Fig. 3D, E). Further, GPC-4 expression is blocked in the presence of NF- $\kappa$ B inhibitor IMD-0354 (Fig. 3G,H). It altogether demonstrates that neurotoxic astrocytes express GPC-4 via NF- $\mathrm{B}$ dependent pathway

\section{Glypican -4 drives APOE4-induced LRP1 trafficking and tau propagation}

The LRP1 (low-density lipoprotein receptor-related protein 1) is involved in tau uptake and spreading ${ }^{38}$. We next determined whether the APOE variants have differential effects on the LRP1 receptor. For this purpose, we monitored the effects of APOE2 and APOE4 on the total and surface LRP1 levels. We found that the addition of APOE2 had no effect on both the total and surface LRP1 levels (Fig. 4A-C). By contrast, APOE4 enhanced the surface LRP1 levels but not the total LRP1 levels (Fig. 4A-C). Further, active exocytic and endocytic pathways are required for APOE4-induced surface LRP1 and APOE2 mediated downregulation of APOE4-induced surface LRP1, respectively (Fig. S3).

We next reasoned that GPC-4 being an astrocyte-secretory factor would interact with neuronal LRP1 to induce tau pathology in neurons. To test this notion, we targeted the likelihood of GPC4-LRP1 interaction and assessed the effect of GPC-4 on the key signal transduction protein. First, we validated the GPC4-LRP1 interaction from human postmortem brain tissue by coimmunoprecipitation (Fig. 4D). In the neuronal culture, the addition of GPC-4 unaltered the total 
LRP1 (Fig. 4E, F), whereas the surface LRP1 levels increased greatly (Fig. 4E, G). This suggests that GPC-4 interacts with LRP1 and regulates trafficking of LRP1.

We next investigated whether APOE4 is dependent on GPC-4 to induce surface LRP1 levels. To investigate this notion, we treated neurons with either APOE4 alone or APOE4 with GPC-4 shRNA (Fig. 4H). The total LRP1 was unaltered (Fig. 4I). However, APOE4 in the presence of GPC-4 shRNA showed a significant reduction of surface LRP1 compared to APOE4 alone (Fig. 4J). Altogether, these data indicate that APOE4 increases surface LRP1 through GPC-4.

\section{Glypican-4 drives APOE4-mediated tau propagation}

Given that both APOE4 and GPC-4 enhanced tau pathology, we next examined whether the differential efficacy of APOE2 and APOE4 is dependent on the GPC-4 interaction. Immunoprecipitation analysis from a human postmortem brain showed that APOE directly interacts with GPC-4 (Fig. S4A). Glycosaminoglycan chains in proteoglycans including in HSPGs play an important role in binding with other proteins. To test whether APOE2 and APOE4 differentially interact with glycosaminoglycans of GPC-4, we incubated a GPC-4-coated Sepharose column with either APOE2 or APOE4 for $1 \mathrm{~h}$ and eluted it with $\mathrm{NaCl}$ gradients. We observed that neither isoforms showed preferential binding with GPC-4 (data not shown). However, we cannot rule out that protein segments rather than the glycan part may bind to the APOE variants differently. To test this, we incubated GPC-4 either with APOE2 or APOE 4 at room temperature for $1 \mathrm{~h}$ as described in Figure S4B and analyzed with a native gel. APOE2+GPC4 combination did not show any major shifts, whereas APOE4+GPC-4 mix showed a robust shift both with GPC-4 and APOE antibodies. Further, treatment with 2-mercaptoethanol disturbed the shift, suggesting that core proteins of GPC-4 and APOE4 are in direct interaction.

Given that GPC_4 strongly interacts with APOE4 and is involved in APOE4-mediated LRP1 trafficking, we next reasoned that GPC-4 would play an important role in APOE4-mediated tau pathology. We treated the primary neuronal culture with APOE4 or APOE4 with GPC-4 shRNA for $24 \mathrm{~h}$ and then incubated it with $1 \mu \mathrm{g} / \mathrm{ml}$ of human tau protein for $1 \mathrm{~h}$. Immunohistochemistry with human tau antibody HT-7 showed that APOE4 increased tau uptake but this was blocked in the presence of GPC-4 shRNA. This result highlights the role of GPC-4 in regulating the APOE4- 
induced tau uptake (Fig. 4K, L). We next investigated the role of APOE4 and GPC-4 in tau propagation using P301S animals (Fig. 4M). We observed APOE4-mediated tau accumulation in the ipsilateral hippocampus (Fig. S5); further, APOE4 induced tau spreading to contralateral hippocampus and cortical regions (Fig. 4N). Interestingly, APOE4-mediated tau pathology was dramatically reduced in the absence of GPC-4 (Fig. 4N, O). This demonstrates that APOE4 induces both tau accumulation and spreading through GPC-4 (Fig. S4C).

\section{GPC-4 drives tau propagation via IRE1 $\alpha$ pathway}

To further investigate the role of GPC-4 in tau pathology in vivo, we injected GPC-4 protein stereotaxically in the hippocampal CA1 region of P301S animals (Fig. 5A). After one week of incubation period, we observed a tremendous accumulation of phosphorylated tau in the CA1 region (Fig. 5B-D). Additionally, injection of GPC-4 in cortical regions as well induced tau accumulation (Fig. S5D).

We next examined a possible molecular mechanism that underlies GPC-4 induced tau pathology. Unfolded protein response (UPR) pathways play a major role in protein misfolding disorders such as $\mathrm{AD}^{39,40}$. IRE1 $\alpha$ is one of the major UPR pathways involve in protein quality control ${ }^{41}$. Phosphorylated IRE1 $\alpha$ (pIRE1 $\alpha$ ) is detected within degenerating pyramidal neurons in AD patients

${ }^{42}$. We therefore tested whether GPC-4 induced tau pathology is associated with IRE1 $\alpha$ pathway. Addition of GPC-4 protein in neuronal culture enhanced phosphorylation of IRE1 $\alpha$ protein (Fig. $5 \mathrm{E}, \mathrm{F})$. We next blocked the IRE1 $\alpha$ pathway with a pharmacological compound, KIRA6, to test whether GPC-4 induced tau pathology can be reversed ${ }^{43}$. We injected GPC-4 protein in P301S animals, i.p injected KIRA6 daily for 3 weeks $(5 \mathrm{mg} / \mathrm{kg})$, and analyzed on contralateral hippocampus for tau propagation (Fig. 5G). Interestingly, GPC-4 induced tau propagation is blocked in KIRA6 treated animals (Fig. 5H, I). It suggests that GPC-4 induces tau pathology via the IRE1 $\alpha$ pathway.

\section{DISCUSSION}

Our study addresses at the molecular level why APOE4 carrying individuals are at risk of developing $\mathrm{AD}$ pathophysiology. We found that APOE4 AD patients have more tau accumulation both inside and outside of neurons, and APOE4 protein induces tau propagation. A subtype of 
neurotoxic astrocyte secreted GPC-4 triggers tau accumulation and propagation. We further demonstrated that APOE4-induced tau uptake and propagation is dependent on GPC-4 protein. We finally showed that GPC-4 induces tau pathology via the IRE1 $\alpha$ pathway.

Human IPSCs-derived APOE4 neurons expressed more pTau ${ }^{44}$. However, human tau PET studies tend to contradict each other ${ }^{10,45,46}$. To our knowledge there are no studies carried out to compare tau accumulation in APOE homozygous variants of human postmortem brain tissues. Our immunohistochemical studies clearly demonstrated the presence of more neurofibrillary tangles, neuropil threads and neuritic plaque in APOE4/4 AD patients. Further insights into how these tau pathologies would function differently in APOE variants is required to understand the disease progression.

An association of APOE4 with tau is independent of amyloid beta levels ${ }^{22,23}$. Our data demonstrating APOE4 mediated tau propagation are in agreement with these studies. Importantly, why APOE4 behaves as an AD risk factor ${ }^{5}$, while APOE2 is protective against $\mathrm{AD}^{8}$ is still unknown. With differential actions of APOE2 and APOE4 in tau pathology, our in vitro studies showed that APOE4-induced tau propagation is reversed by APOE2 protein. Furthermore, APOE4 enhanced the surface expression of LRP1, but the same effect was reversed in the presence of APOE2. Interestingly, compared to APOE2, APOE4 showed a strong interaction with GPC-4, suggesting that the molecular architecture of APOE4 carrying individuals possibly determine the onset of AD pathologies. However, it is still possible the geographic regions, race or environmental factors might play an important role in APOE4-AD risks. For example, APOE4-carrying European descents are at higher risk of developing AD compared to African-Americans or Hispanic populations ${ }^{2}$. Therefore, to further understand an association of APOE4 with AD, molecular studies at the level of geographic region or race would be required.

Activated A1 astrocytes are considered as neurotoxic astrocytes whose secretory molecules may be involved in worsening of $\mathrm{AD}$ pathology $\mathrm{y}^{37,47}$. Interestingly, we found that GPC-4 is mainly expressed within a neurotoxic astrocytic population and astrocytes-derived GPC-4 induces tau pathology. Proteostasis is vital for cellular protein quality control. Altered proteostasis leads to cellular stress, aging, increased protein half-life, protein misfolding and more deleterious effects 
39,48. Activation of GPC-4 mediated IRE1 $\alpha$ pathway suggests that GPC-4 alters the normal proteostasis process. Further, in the absence of GPC-4, APOE4 failed to induce tau accumulation and spreading. It supports the idea that APOE4 is neither necessary nor sufficient in development of $\mathrm{AD}$, but potentially other cofactors such as GPC-4 would be needed ${ }^{49}$.

In conclusion, our results reveal that astrocyte secreted GPC-4 drives tau pathology. Moreover, GPC-4 mediates APOE-4 dependent LRP1 trafficking and tau pathology. Further, IRE1 $\alpha$ pathway is needed for GPC-4 induced tau pathology. Thereby, our study provides a mechanism which explains why APOE4 carriers are at risk in developing AD pathogenesis and a way to intervene the tau spread.

\section{Acknowledgments}

This work was supported by funding from NIH R01 AG063819 (ACP), NIH R01AG064020 (ACP), Paul B. Beeson Emerging Leaders Career Development Award in Aging K76 AG054772 (ACP), the BrightFocus Foundation (ACP), the DANA Foundation (ACP), the Alzheimer's Drug Discovery Foundation (ACP), the Alzheimer's Association (ACP), the Bernard L. Schwartz Award for Physician Scientist (ACP). We thank Jill Gregory for generating graphical representation, Mount Sinai Brain Bank for providing human brain samples, and Patrick Hof, Alison Goate and Sam Gandy for insightful comments. We thank Kirill Gorbachev for assisting with quantification analysis.

\section{The authors have no conflict of interest.}

\section{References}

1. Mahley, R. W. Apolipoprotein E : Cholesterol Transport. Science (80-. ). 240, 622-630 (1988).

2. Belloy, M. E., Napolioni, V. \& Greicius, M. D. A Quarter Century of APOE and Alzheimer's Disease: Progress to Date and the Path Forward. Neuron 101, 820-838 (2019).

3. Lane-Donovan, C. \& Herz, J. ApoE, ApoE Receptors, and the Synapse in Alzheimer's 
Disease. Trends Endocrinol. Metab. 28, 273-284 (2017).

4. Weisgraber, K. H., Rall, S. C. \& Mahley, R. W. Human E Apoprotein Heterogeneit. J. Biol. Chem. 256, 9077-9083 (1981).

5. Liddell, M., Williams, J., Bayer, A., Kaiser, F. \& Owen, M. Confirmation of association between the e4 allele of apolipoprotein E and Alzheimer's disease. J. Med. Genet. 31, 197-200 (1994).

6. Saunders, A. M. Association of apolipoprotein E allele $\varepsilon 4$ with late-onset sporadic Alzheimer's disease. Neurology 43, 1467 (1993).

7. Strittmatter, W. J. et al. Apolipoprotein E: High-avidity binding to $\beta$-amyloid and increased frequency of type 4 allele in late-onset familial Alzheimer disease. Proc. Natl. Acad. Sci. U. S. A. 90, 1977-1981 (1993).

8. Corder, E. H. et al. Protective effect of apolipoprotein E type 2 allele for late onset Alzheimer disease. Nat. Genet. 7, 180-184 (1994).

9. Pericak-Vance, M. A. et al. Linkage studies in familial Alzheimer disease: Evidence for chromosome 19 linkage. Am. J. Hum. Genet. 48, 1034-1050 (1991).

10. Montagne, A. et al. APOE4 leads to blood-brain barrier dysfunction predicting cognitive decline. Nature 581, 1-6 (2020).

11. Iqbal, K. et al. Protein changes in senile dementia. Brain Res. 77, 337-343 (1974).

12. Masters, C. L. et al. Amyloid plaque core protein in Alzheimer disease and Down syndrome. Proc. Natl. Acad. Sci. U. S. A. 82, 4245-4249 (1985).

13. Li, C. \& Götz, J. Tau-based therapies in neurodegeneration: Opportunities and challenges. Nat. Rev. Drug Discov. 16, 863-883 (2017).

14. Arboleda-Velasquez, J. F. et al. Resistance to autosomal dominant Alzheimer's disease in an APOE3 Christchurch homozygote: a case report. Nat. Med. 25, 1680-1683 (2019).

15. Kolb, H. C. \& Andrés, J. I. Tau positron emission tomography imaging. Cold Spring Harb. Perspect. Biol. 9, 1-18 (2017).

16. Hanseeuw, B. J. et al. Association of Amyloid and Tau with Cognition in Preclinical Alzheimer Disease: A Longitudinal Study. JAMA Neurol. 76, 915-924 (2019).

17. Ziontz, J. et al. Tau pathology in cognitively normal older adults. Alzheimer's Dement. Diagnosis, Assess. Dis. Monit. 11, 637-645 (2019).

18. Joie, R. La et al. Prospective longitudinal atrophy in Alzheimer's disease correlates with 
the intensity and topography of baseline tau-PET. Sci. Transl. Med. 12, 1-13 (2020).

19. Agosta, F. et al. Apolipoprotein E $\varepsilon 4$ is associated with disease-specific effects on brain atrophy in Alzheimer's disease and frontotemporal dementia. Proc. Natl. Acad. Sci. U. S. A. 106, 2018-2022 (2009).

20. Ossenkoppele, R. et al. Tau PET patterns mirror clinical and neuroanatomical variability in Alzheimer's disease. Brain 139, 1551-1567 (2016).

21. Hohman, T. J. et al. Sex-specific association of apolipoprotein e with cerebrospinal fluid levels of tau. JAMA Neurol. 75, 989-998 (2018).

22. Shi, Y. et al. ApoE4 markedly exacerbates tau-mediated neurodegeneration in a mouse model of tauopathy. Nature 549, 523-527 (2017).

23. Therriault, J. et al. Association of Apolipoprotein e $\epsilon 4$ with Medial Temporal Tau Independent of Amyloid- $\beta$. JAMA Neurol. 1-10 (2019). doi:10.1001/jamaneurol.2019.4421

24. Dujardin, S. et al. Tau molecular diversity contributes to clinical heterogeneity in Alzheimer's disease. Nat. Med. (2020). doi:10.1038/s41591-020-0938-9

25. Arakhamia, T. et al. Posttranslational Modifications Mediate the Structural Diversity of Tauopathy Strains. Cell 180, 633-644.e12 (2020).

26. Braak, H. \& Del Tredici-Braak, K. Alzheimer's Disease, Neural Basis of. Int. Encycl. Soc. Behav. Sci. Second Ed. 591-596 (2015). doi:10.1016/B978-0-08-097086-8.55001-6

27. Rawat, V. et al. ApoE4 Alters ABCA1 Membrane Trafficking in Astrocytes. J. Neurosci. 39, 9611-9622 (2019).

28. Kim, J. et al. Microrna-33 regulates apoe lipidation and amyloid- $\beta$ metabolism in the brain. J. Neurosci. 35, 14717-14726 (2015).

29. $\mathrm{Hu}, \mathrm{J}$. et al. Opposing effects of viral mediated brain expression of apolipoprotein E2 (apoE2) and apoE4 on apoE lipidation and $\mathrm{A} \beta$ metabolism in apoE4-targeted replacement mice. Mol. Neurodegener. 10, 1-11 (2015).

30. Huang, Y. et al. Apolipoprotein E fragments present in Alzheimer's disease brains induce neurofibrillary tangle-like intracellular inclusions in neurons. Proc. Natl. Acad. Sci. U. S. A. 98, 8838-8843 (2001).

31. Huang, W. A., Zhou, B., Wernig, M. \& Südhof, T. C. ApoE2, ApoE3, and ApoE4 Differentially Stimulate APP Transcription and A $\beta$ Secretion. Cell 168, 427-441.e21 
(2017).

32. Asai, H. et al. Depletion of microglia and inhibition of exosome synthesis halt tau propagation. Nat. Neurosci. 18, 1584-1593 (2015).

33. Allen, N. J. et al. Astrocyte glypicans 4 and 6 promote formation of excitatory synapses via GluA1 AMPA receptors. Nature 486, 410-4 (2012).

34. Zhou, Y. et al. Human and mouse single-nucleus transcriptomics reveal TREM2dependent and TREM2-independent cellular responses in Alzheimer's disease. Nat. Med. 26, 131-142 (2020).

35. Grubman, A. et al. A single-cell atlas of entorhinal cortex from individuals with Alzheimer's disease reveals cell-type-specific gene expression regulation. Nat. Neurosci. 22, 2087-2097 (2019).

36. Habib, N. et al. Disease-associated astrocytes in Alzheimer's disease and aging. Nat. Neurosci. 23, 701-706 (2020).

37. Liddelow, S. A. et al. Neurotoxic reactive astrocytes are induced by activated microglia. Nature 541, 481-487 (2017).

38. Rauch, J. N. et al. LRP1 is a master regulator of tau uptake and spread. Nature 1, (2020).

39. Kaushik, S. \& Cuervo, A. M. Proteostasis and aging. Nat. Med. 21, 1406-1415 (2015).

40. Hetz, C. \& Saxena, S. ER stress and the unfolded protein response in neurodegeneration. Nat. Rev. Neurol. 13, 477-491 (2017).

41. Wolff, S., Weissman, J. S. \& Dillin, A. Differential scales of protein quality control. Cell 157, 52-64 (2014).

42. Hoozemans, J. J. M. et al. The unfolded protein response is activated in pretangle neurons in alzheimer's disease hippocampus. Am. J. Pathol. 174, 1241-1251 (2009).

43. Ghosh, R. et al. Allosteric inhibition of the IRE1 $\alpha$ RNase preserves cell viability and function during endoplasmic reticulum stress. Cell 158, 534-548 (2014).

44. Lin, Y. T. et al. APOE4 Causes Widespread Molecular and Cellular Alterations Associated with Alzheimer's Disease Phenotypes in Human iPSC-Derived Brain Cell Types. Neuron 98, 1141-1154.e7 (2018).

45. Ossenkoppele, R. et al. Assessment of Demographic, Genetic, and Imaging Variables Associated with Brain Resilience and Cognitive Resilience to Pathological Tau in Patients with Alzheimer Disease. JAMA Neurol. 1-11 (2020). doi:10.1001/jamaneurol.2019.5154 
46. Mattsson, N. et al. Greater tau load and reduced cortical thickness in APOE $\varepsilon 4$-negative Alzheimer's disease: a cohort study. Alzheimer's Res. Ther. 10, 1-12 (2018).

47. Khakh, B. S. \& Deneen, B. The Emerging Nature of Astrocyte Diversity. Annu. Rev. Neurosci. 42, 187-207 (2019).

48. McShane, E. et al. Kinetic Analysis of Protein Stability Reveals Age-Dependent Degradation. Cell 167, 803-815.e21 (2016).

49. Stemmer, W. P. C. APOE*4-associated Alzheimer's disease risk is modified by alpha 1antichymotrypsin polymorphism. Nat. Genet. 13, 4-6 (1995).

50. Risher, W. C. et al. Astrocytes refine cortical connectivity at dendritic spines. Elife 3, 124 (2014).

Figure legends

Figure 1. APOE4 AD patients have more tau accumulation and APOE4 enhance tau propagation. A) Representative IHC images of postmortem tissues from APOE2/3(control), APOE2/2(AD), APOE3/3 (AD) and APOE4/4 (AD) individuals stained with AT8 and aceTau (Lys174) antibodies ( $\mathrm{n}=5-7)$. Note the presence of Neurofibrillary tangles (arrow), neuropil threads (asterisk) and neuritic plaque (arrowhead). B) Compared to APOE3/3 and APOE2/2, APOE4/4 shows presence of more neurofibrillary tangle-containing neurons. C) Representative IHC staining of APOE treated neuronal culture (Tau P301S* Tau KO animals) shows that APOE 4 treatments enhanced tau spreading whereas APOE2 significantly reduced APOE4-mediated tau spreading (D). $\mathrm{n}=3-4$, one-way ANOVA, mean \pm SEM, IHC scale bars $=20 \mu \mathrm{m}$. **P<0.01, $* * * \mathrm{P}<0.001$ and $* * * * \mathrm{P}<0.0001$

Figure 2. Astrocyte -secreted Glypican-4 drives tau pathology

A) Western blot analysis of tau protein from neuronal culture (Tau P301S animals) treated with GPC-4 protein shows that GPC-4 significantly enhanced pTau levels (C) whereas no changes observed in total tau protein (B). D) Representative IHC staining of GPC-4 treated neuronal culture (Tau P301S animals) with AT8, MAP2 and DAPI demonstrates that GPC-4 treatment enhanced AT8 level in neurons (E). F) Representative IHC staining of GPC-4 treated neuronal culture (Tau P301S* Tau KO animals) shows that GPC-4 treatment enhances tau spreading (G). H) Schematic 
diagram shows that astrocyte-conditioned media (ACM) from astrocyte culture was added to neuronal culture. I-K) Addition of ACM to neuronal culture increased AT8 levels whereas total tau was unaltered. L) Schematic diagram shows that astrocytes were treated with GPC-4 shRNA and the resulting GPC-4 deprived ACM was added to neuronal culture. M-O) Addition of GPC-4 deprived ACM failed to induce tau phosphorylation in neurons. $n=3-4$, unpaired Student's t-test, mean \pm SEM, IHC scale bars $=20 \mu \mathrm{m} . * \mathrm{P}<0.05, * * \mathrm{P}<0.01$, and $* * * \mathrm{P}<0.001$

\section{Figure 3. GPC-4 is expressed in a subtype of neurotoxic astrocytes.}

A) Western blot analysis from APOE44-carrying AD patients and APOE4-noncarrying AD patients show that GPC-4 is up-regulated in APOE4 carrying individuals. B) Immunohistochemistry from the human brain shows that GPC-4 is expressed in GFAP astrocytes. C) Heat map shows that GPC-4 is enriched in AD-Associated astrocytes. D, E) Western blot analysis from astrocyte culture shows that treatment with TNF- $\alpha$ and IL-1 $\beta$ significantly increased expression of GPC-4 and activated NF- $\kappa$ B pathway. G, H) Western blot analysis from astrocyte

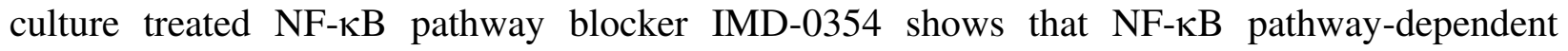
expression of GPC-4. $\mathrm{n}=3-4$, unpaired Student's t-test, mean $\pm \mathrm{SEM}, * * \mathrm{P}<0.01, * * * \mathrm{P}<0.001$ and $* * * * \mathrm{P}<0.0001$. IHC scale bars $=20 \mu \mathrm{m}$.

\section{Figure 4. GPC-4 is required for APOE 4-mediated LRP1 trafficking and tau pathology}

A-C) Western blot analysis from primary neuronal culture treated with APOE proteins show that APOE4 significantly enhances surface LRP1 (S.LRP1) (B). There were no changes in total LRP1 (T.LRP1) (C). D) Immunoprecipitation of human postmortem brain protein samples with LRP1 antibody and subsequent western blotting revealed that LRP1 and GPC-4 are in the same complex. E-G) Western blot analysis from neuronal culture treated with GPC-4 protein shows that GPC-4 significantly enhanced trafficking of surface LRP1 levels $(\mathrm{G})$, whereas total LRP1 levels were not affected (F). H-J) Western blot analysis from neuronal culture treated with APOE4 and with GPC-4 shRNA shows that APOE4 induced surface expression of LRP1 is mediated by GPC-4. The GPC-4 shRNA treatment reduced the surface expression of LRP1 in the presence of APOE4 compared to APOE4 alone (J). K) Representative IHC staining of APOE4 treated neuronal culture shows that APOE4 treatment enhanced tau uptake and presence of GPC-4 shRNA did the converse, suggesting that GPC-4 is required for APOE4-mediated tau uptake (L). C) A unilateral 
injection of APOE4 or GPC-4 shRNA in the CA1 region of P301S animals. N, O) APOE4 protein induced tau tau spreading to contralateral hippocampus. However, the presence of GPC-4 shRNA halted APOE 4 mediated tau accumulation and spreading. $n=3-5$, one-way ANOVA, mean $\pm \mathrm{SEM}$, IHC scale bars $=20 \mu \mathrm{m}(\mathrm{C})$ or $100 \mu \mathrm{m}(\mathrm{E}) . * * \mathrm{P}<0.01$ and $* * * * \mathrm{P}<0.0001$.

Figure 5. Inhibition of IRE1 $\alpha$ pathway blocks GPC-4 mediated tau propagation.

A) A unilateral injection of GPC-4 protein in the CA1 region of P301S animals and analysis after a week. B-D) After one week of incubation period, elevated levels of tau accumulation were observed in GPC-4 injected hippocampal regions. E) Cultured primary neurons were treated with GPC-4 for $1 \mathrm{hr}$ and performed a western blotting. GPC-4 treatment shows a significant activation of IRE1 $\alpha$ pathway (F). G) A unilateral injection of GPC-4 protein in the CA1 region of P301S animals and daily ip injection IRE1 $\alpha$ pathway inhibitor KIRA6 for 3 weeks. H, I) After 3 weeks, GPC-4 induced tau propagation on contralateral sites and KIRA6 treatment blocked GPC-4 induced tau propagation. . $\mathrm{n}=3-5$, unpaired Student's t-test, mean $\pm \mathrm{SEM}, * * \mathrm{P}<0.01$, $* * * \mathrm{P}<0.001$ and $* * * * \mathrm{P}<0.0001$. IHC scale bars $=20 \mu \mathrm{m}$ and $100 \mu \mathrm{m}$. 
Figure 1

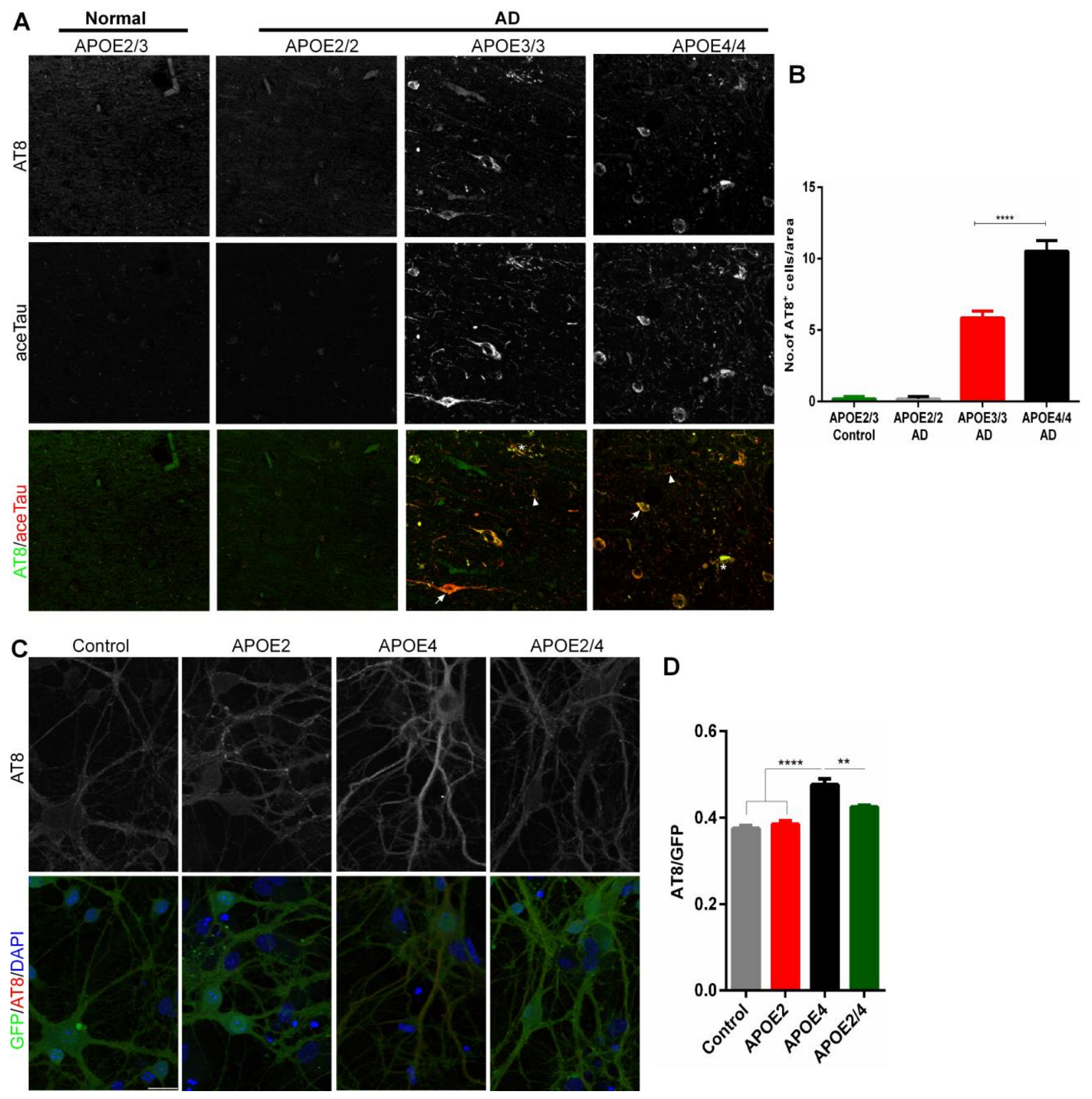


Figure 2
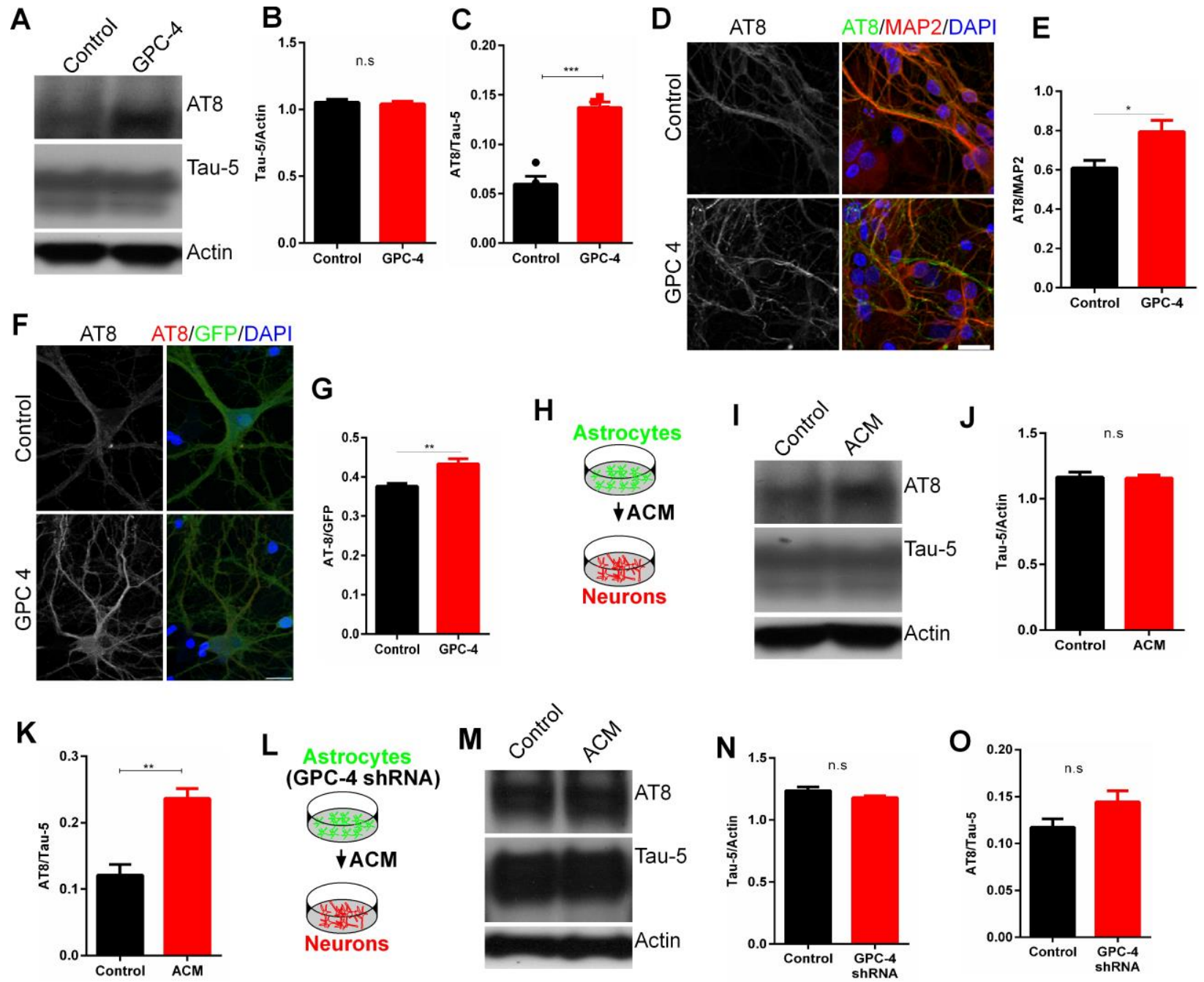

\section{H}
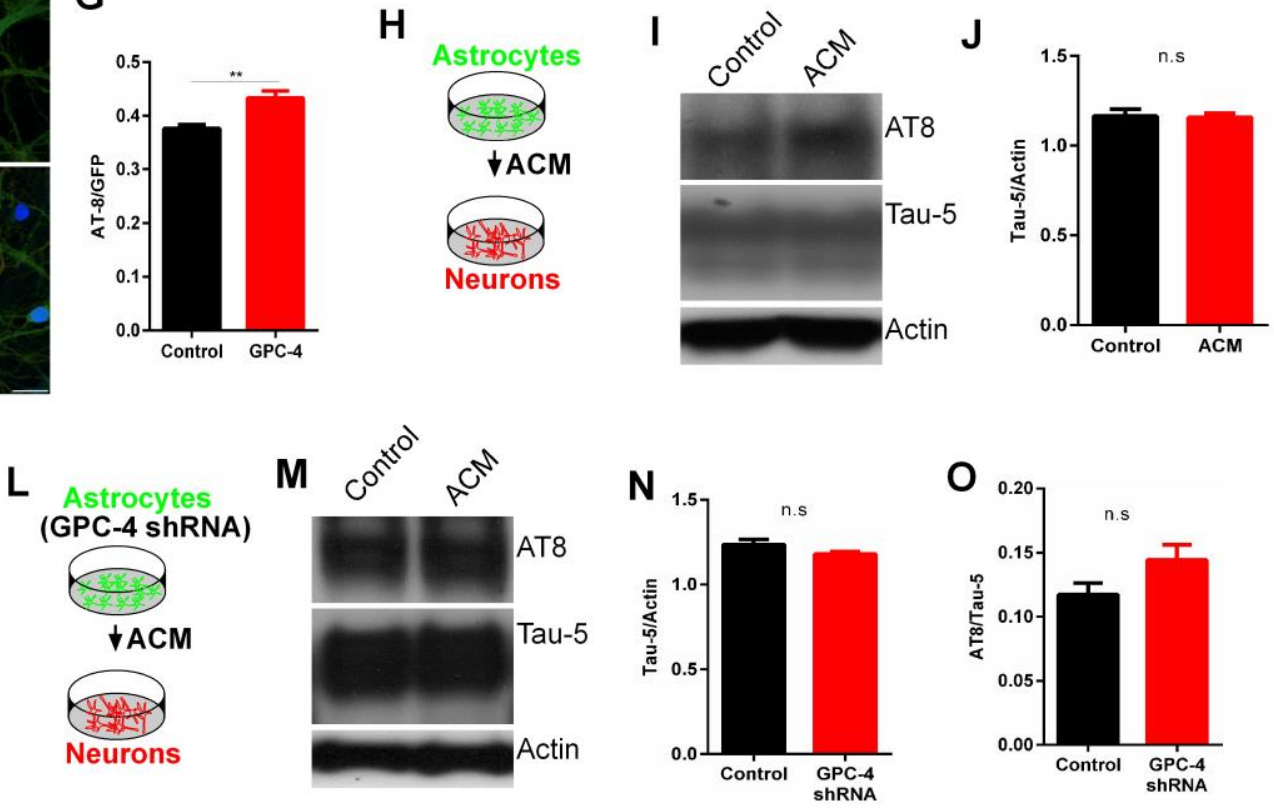
Figure 3

A
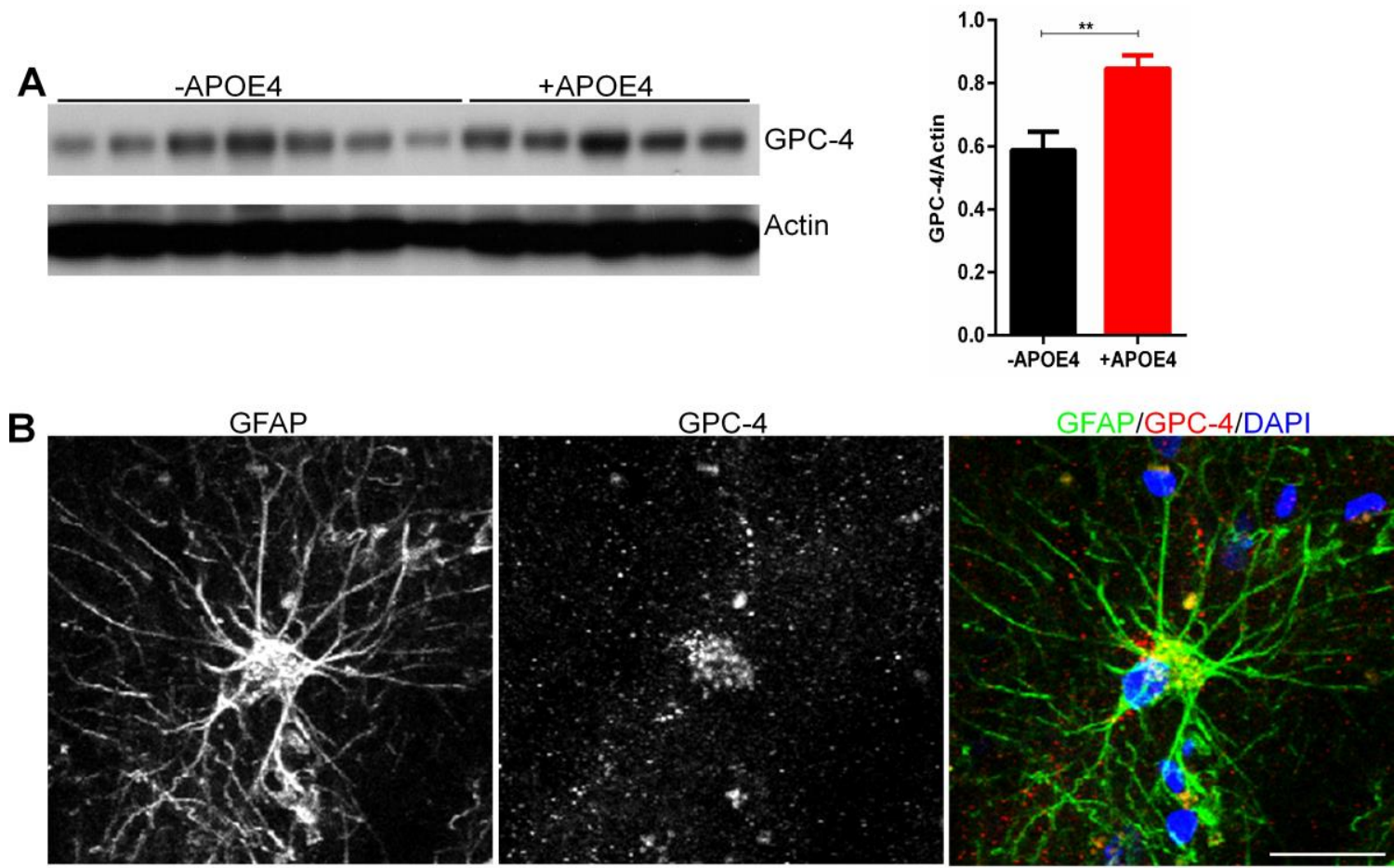

C

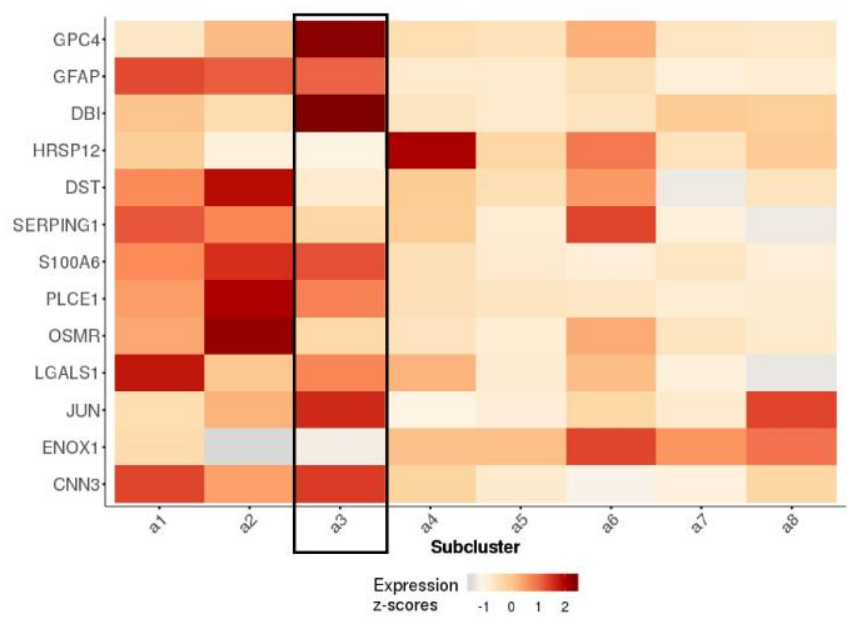

D
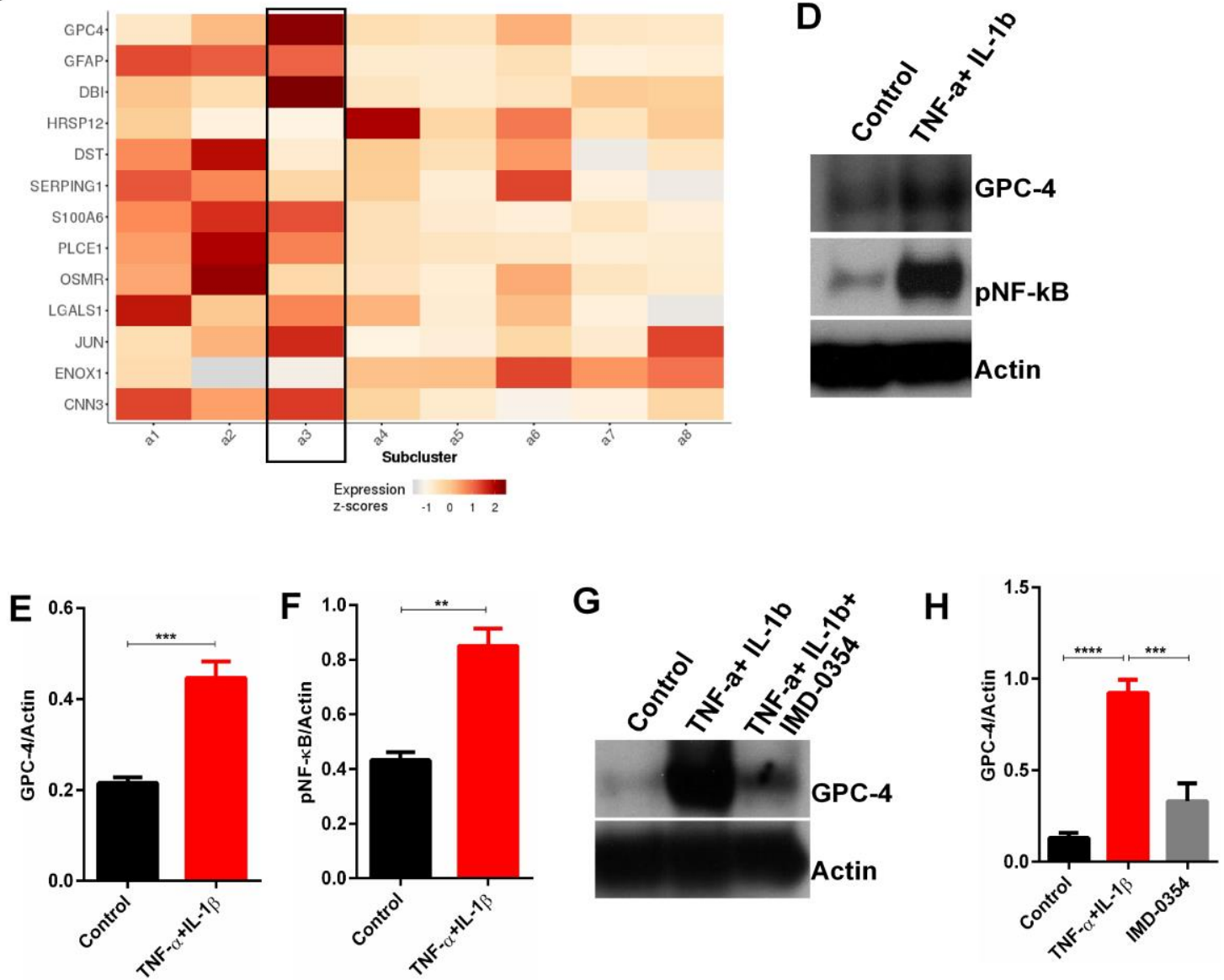
Figure 4
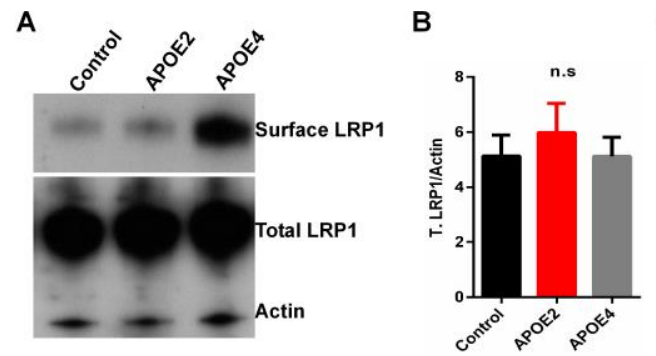

B
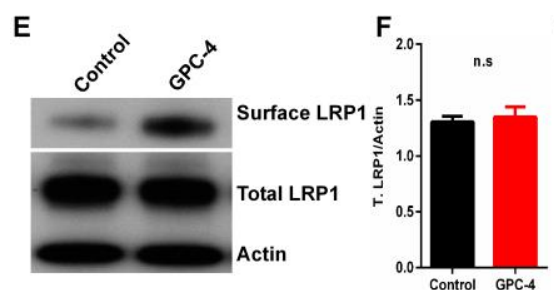

G
C

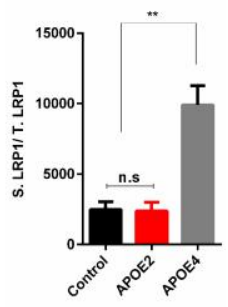

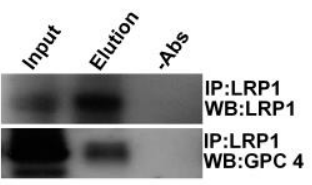

K
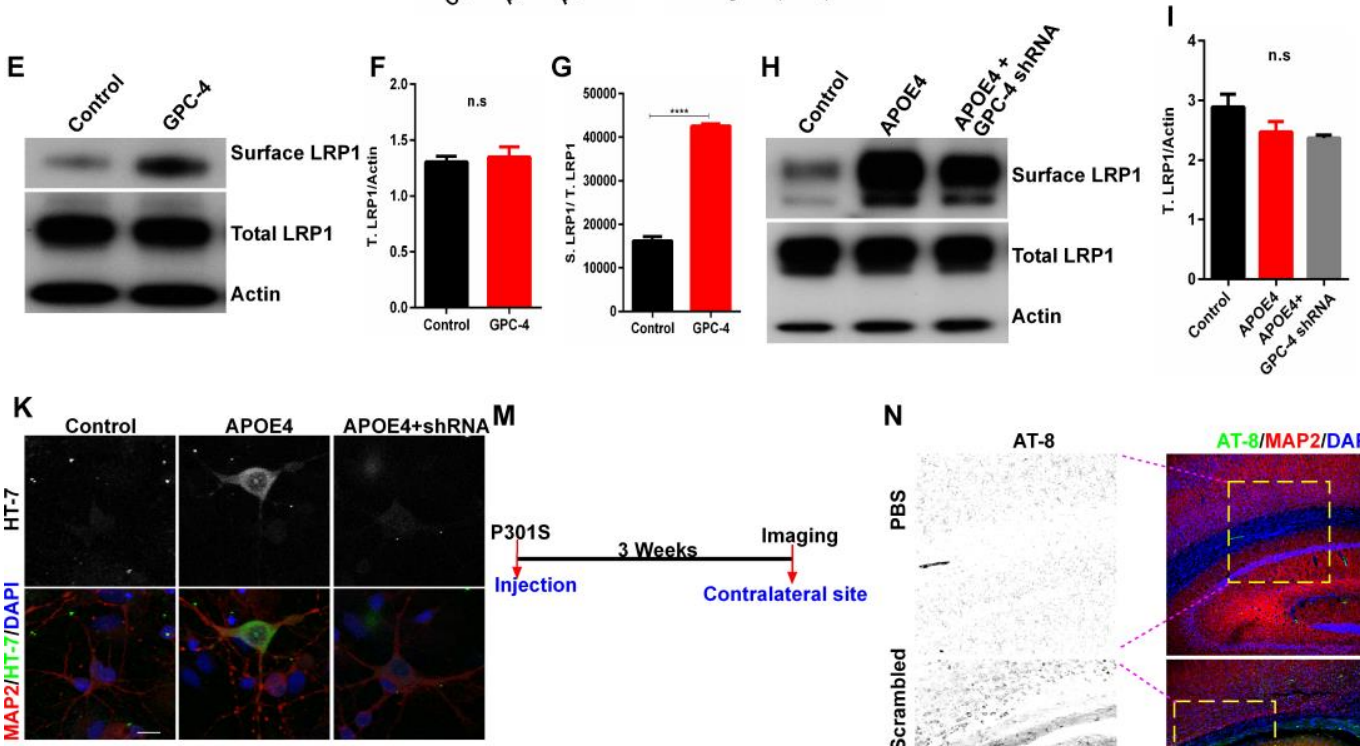

L

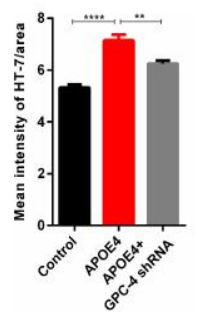

兽

总

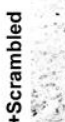

है

AT-8

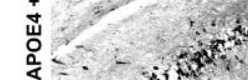

si
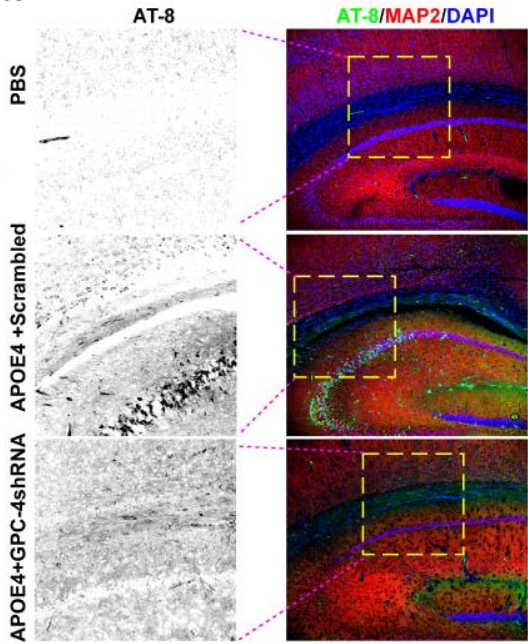

0

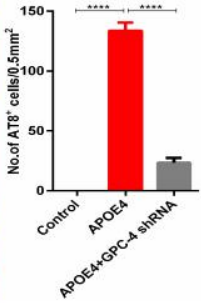


Figure 5

A

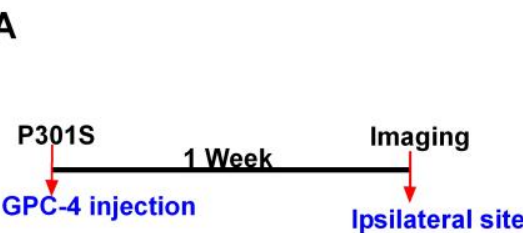

B

Control

GPC-4

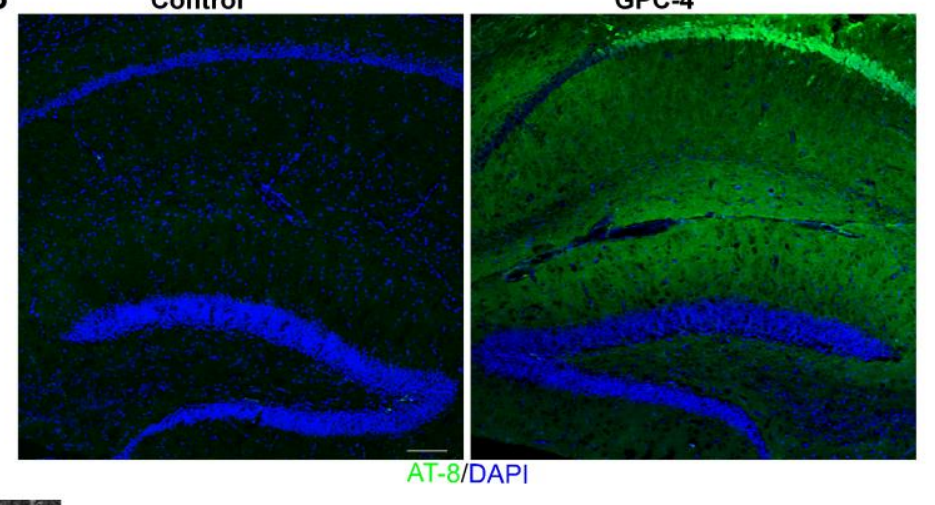

C

Control

GPC-4

E

F

$\stackrel{\infty}{\stackrel{1}{<}}$

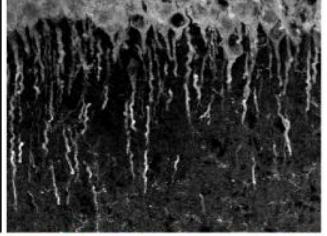

D
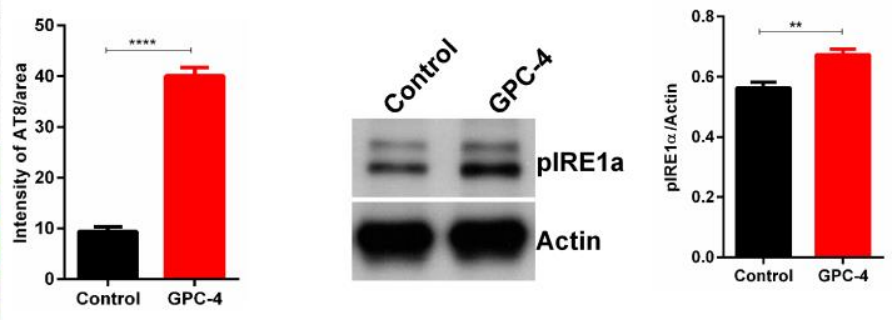

G

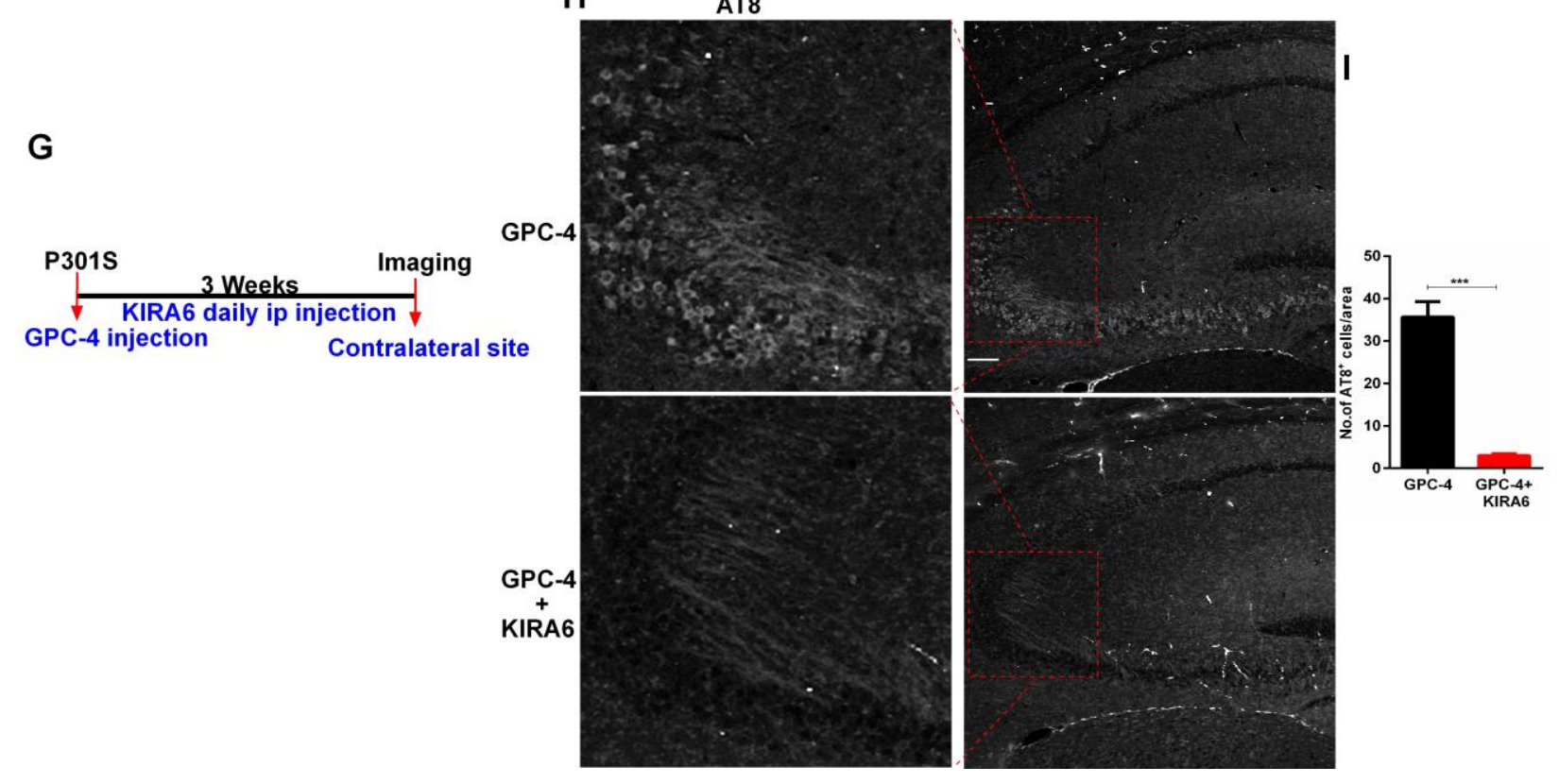

\section{H}

AT8 


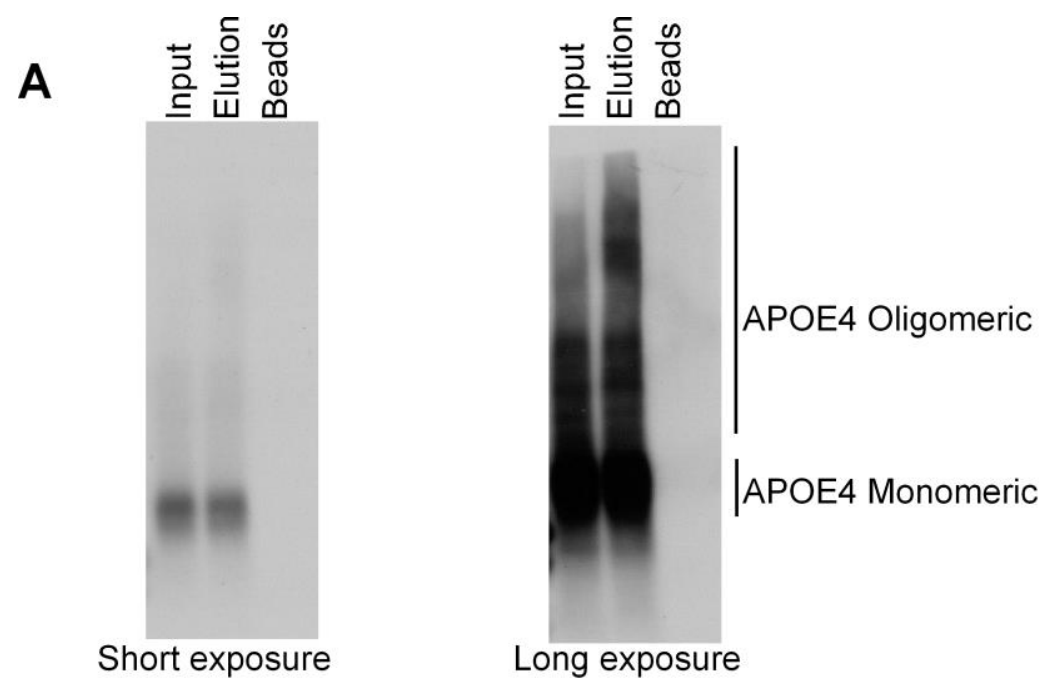

B
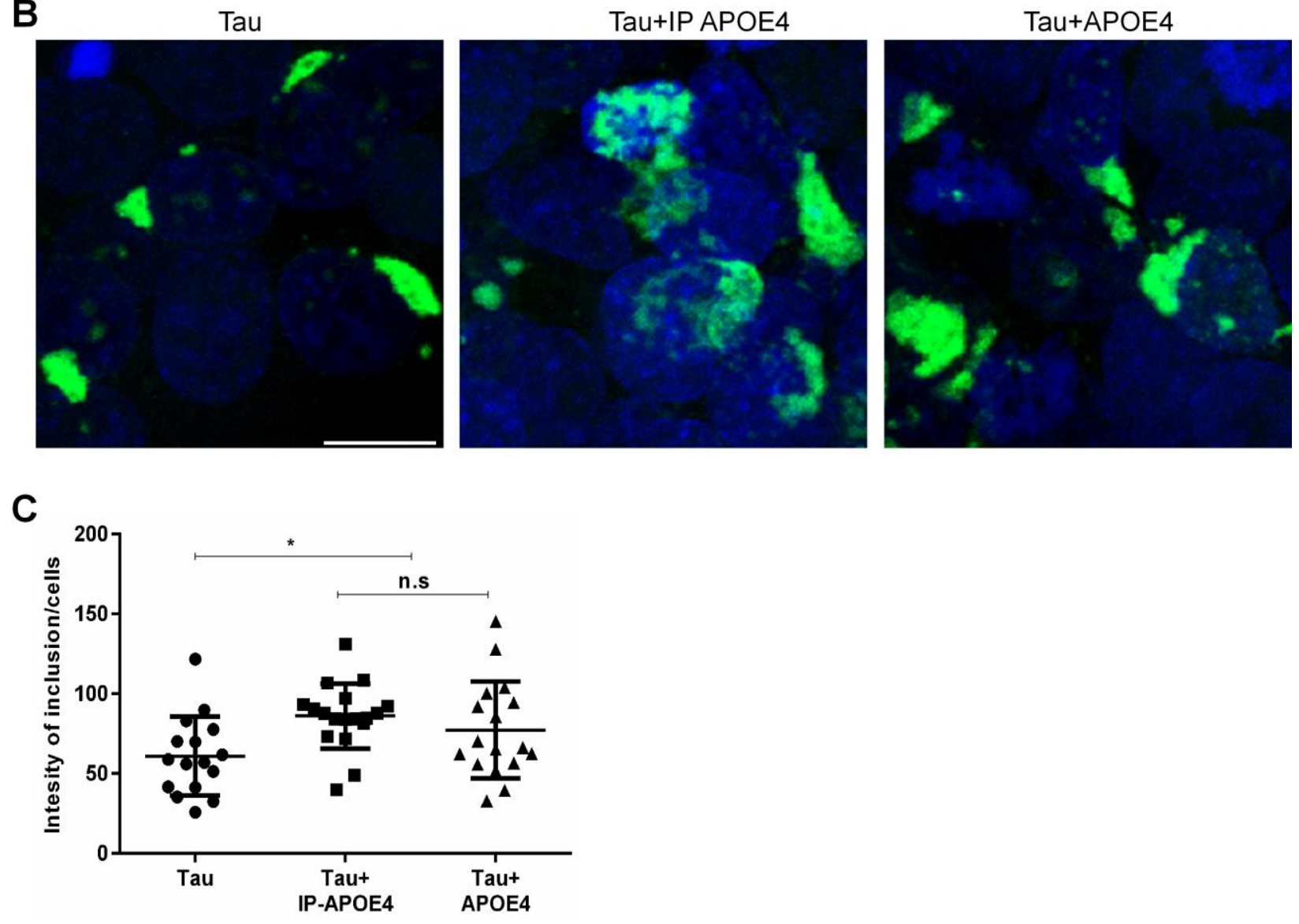

\section{Supplementary Figure 1}

A) Immunoprecipitation with APOE antibodies from APOE44 human brains shows presence of abundant monomeric APOE4 protein. B, C) Tau seeding assay shows that purified APOE4 and purified APOE4-contaning particles induce tau seeding in a similar way. Blue-DAPI. Green-YFP. one-way ANOVA, mean \pm SEM, IHC scale bars $=10 \mu \mathrm{m}$. $* \mathrm{P}<0.05$. 

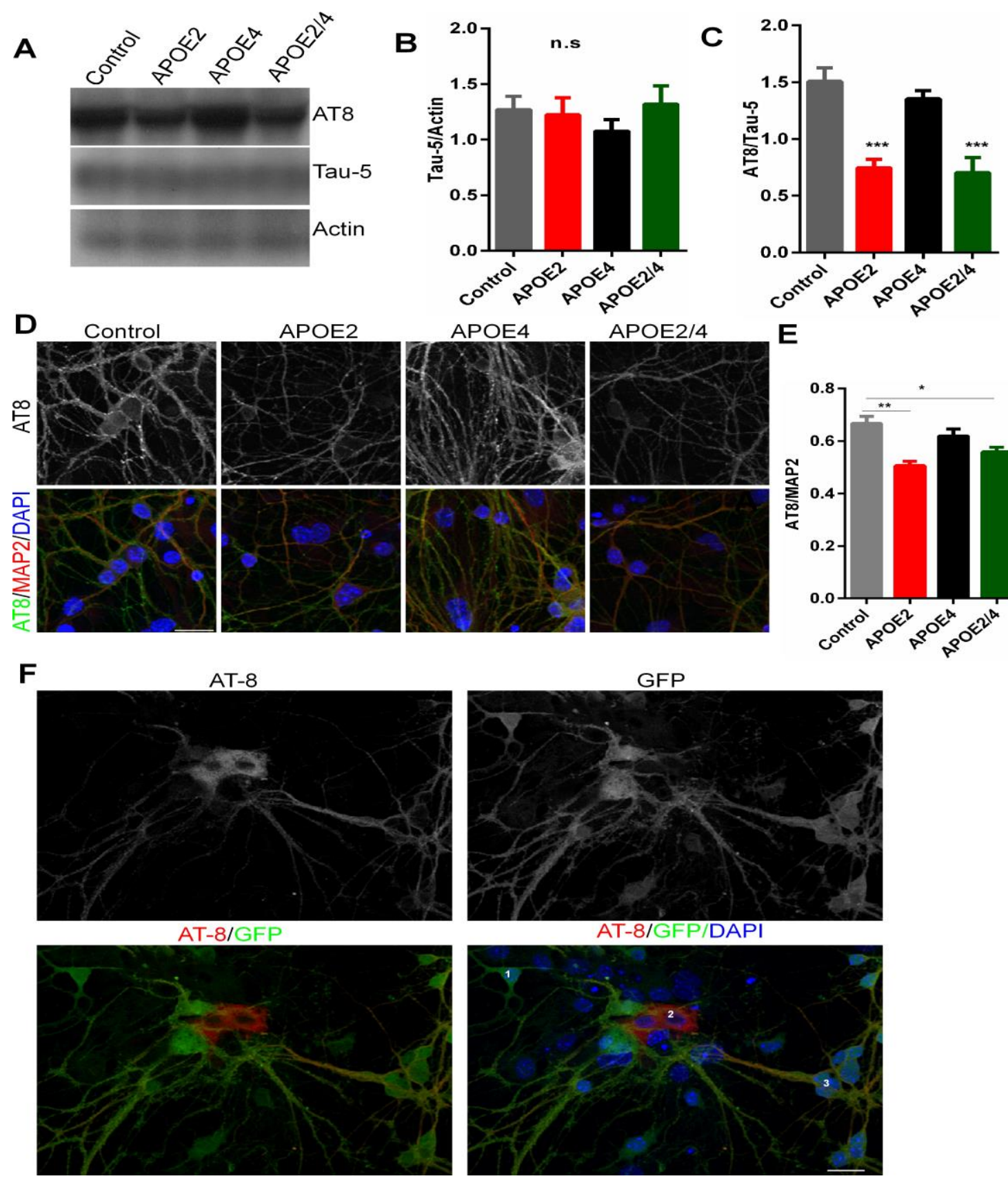

\section{Supplementary Figure 2}

A) Western blot analysis of tau protein from primary neuronal culture (Tau P301S animals) treated with recombinant human APOE proteins show that APOE2 significantly reduced AT8 levels compared to controls and APOE4 treated cells (C). Total tau protein was unaltered (B). D) Representative IHC staining of APOE treated neuronal culture (tau P301S animals) with AT8, MAP2 and DAPI staining demonstrate that APOE2 treatments reduced AT8 levels in neurons (E). F) Representative IHC staining of neuronal coculture from tau P301S* and Tau KO (GFP) animals show that tau protein (AT8) transferred from tau P301S neurons Tau KO GFP neurons. $\mathrm{n}=3-4$, one-way ANOVA, mean \pm SEM, IHC scale bars $=20 \mu \mathrm{m}$. $* \mathrm{P}<0.05$, $* * \mathrm{P}<0.01$ and $* * * \mathrm{P}<0.001$. 
A

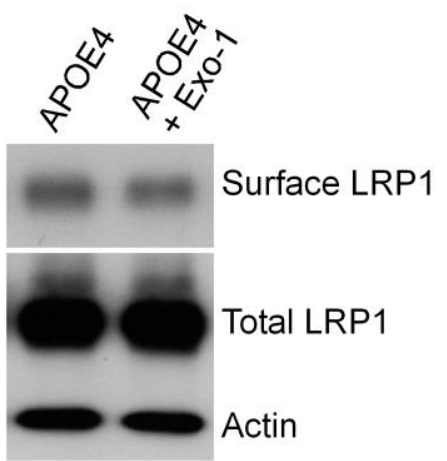

B

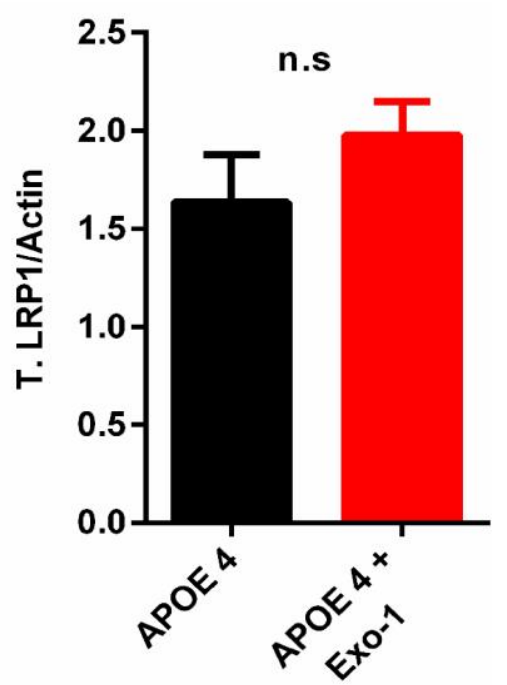

D

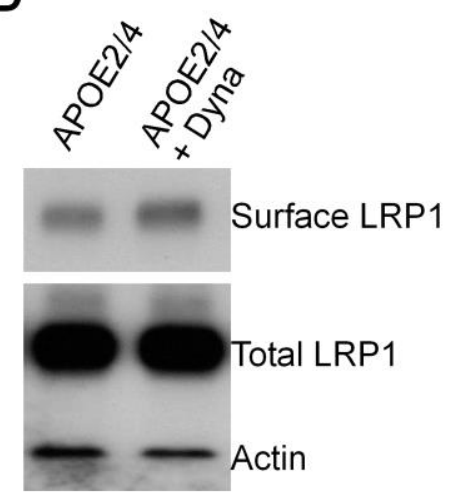

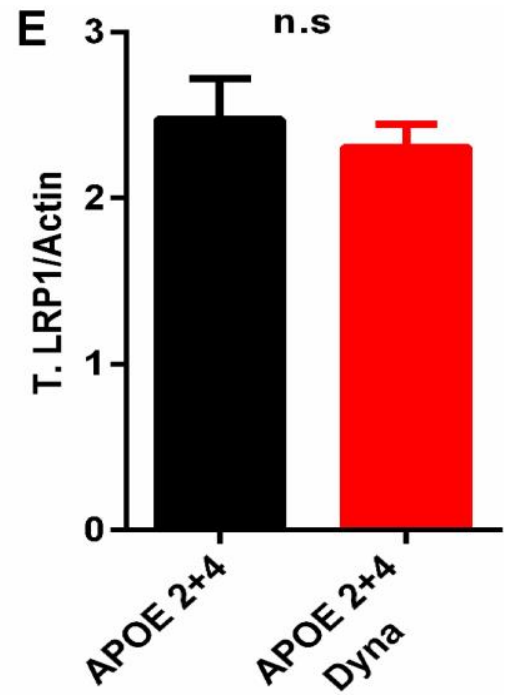

C
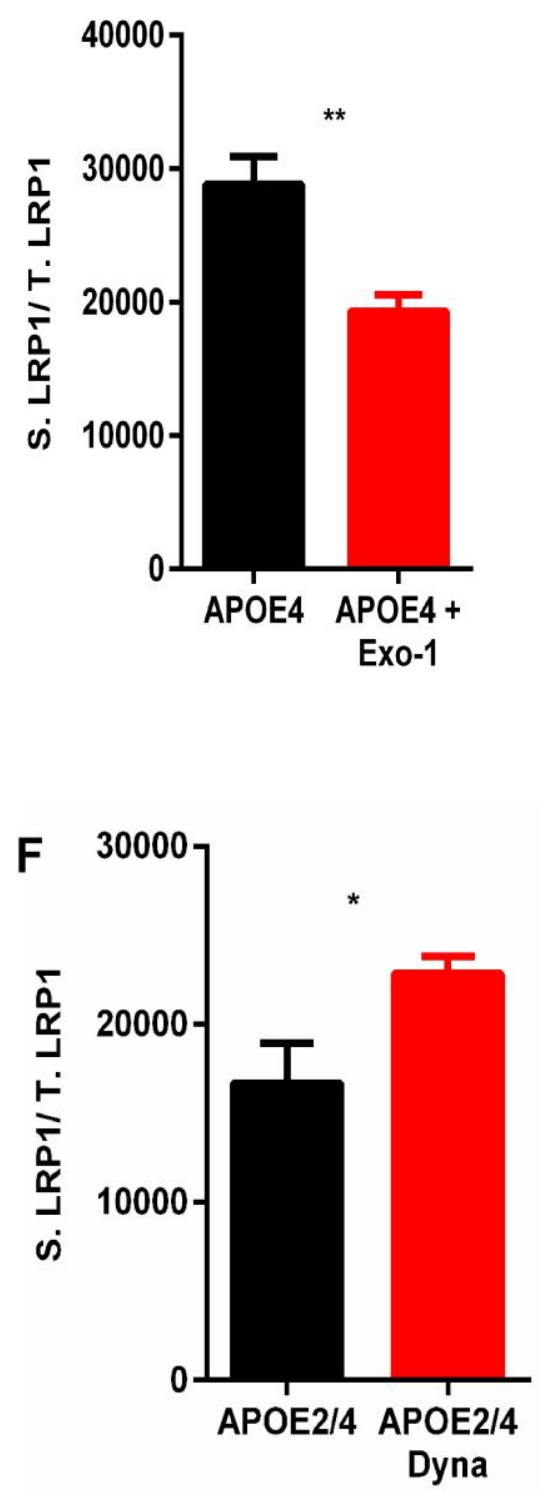

Supplementary Figure 3

A) Western blot analysis from neuronal culture treated with APOE 4 or with exocytosis inhibitor Exo-1 shows that exocytosis is required for APOE4 to mediate surface expression of LRP1 (B, C). D) Western blot analysis from neuronal culture treated with APOE2/4 or with endocytosis inhibitor, Dynasore, shows that APOE2 down-regulated APOE4-mediated surface LRP1 via endocytosis (E, F). $\mathrm{n}=3$ unpaired Student's t-test, mean \pm SEM. $* \mathrm{P}<0.05$ and $* * \mathrm{P}<0.01$. 


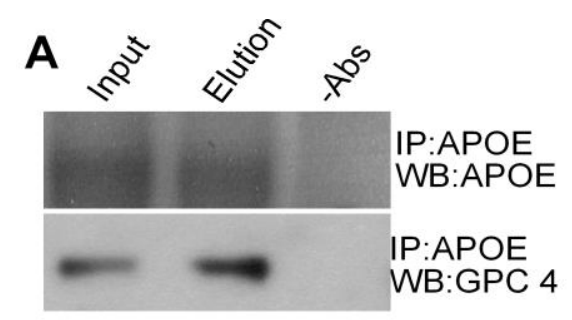

\section{B}

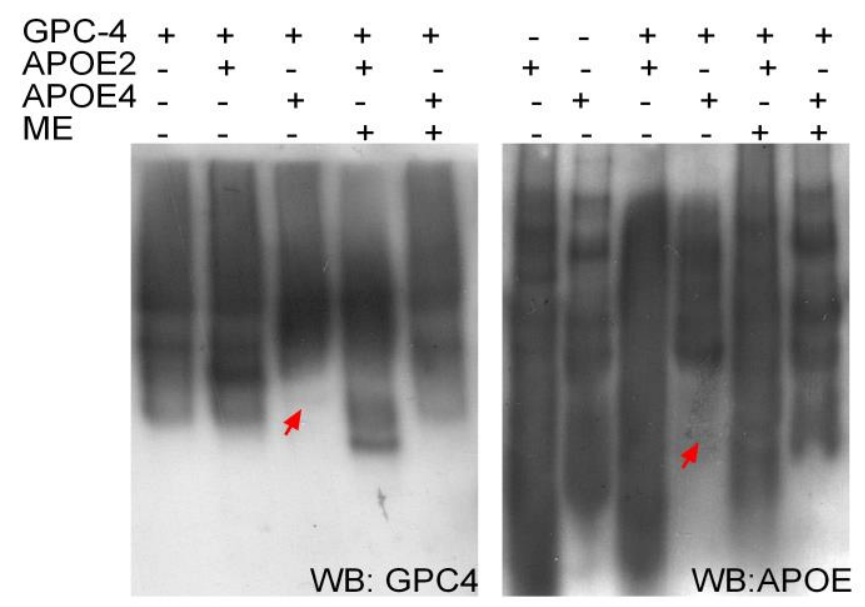

C

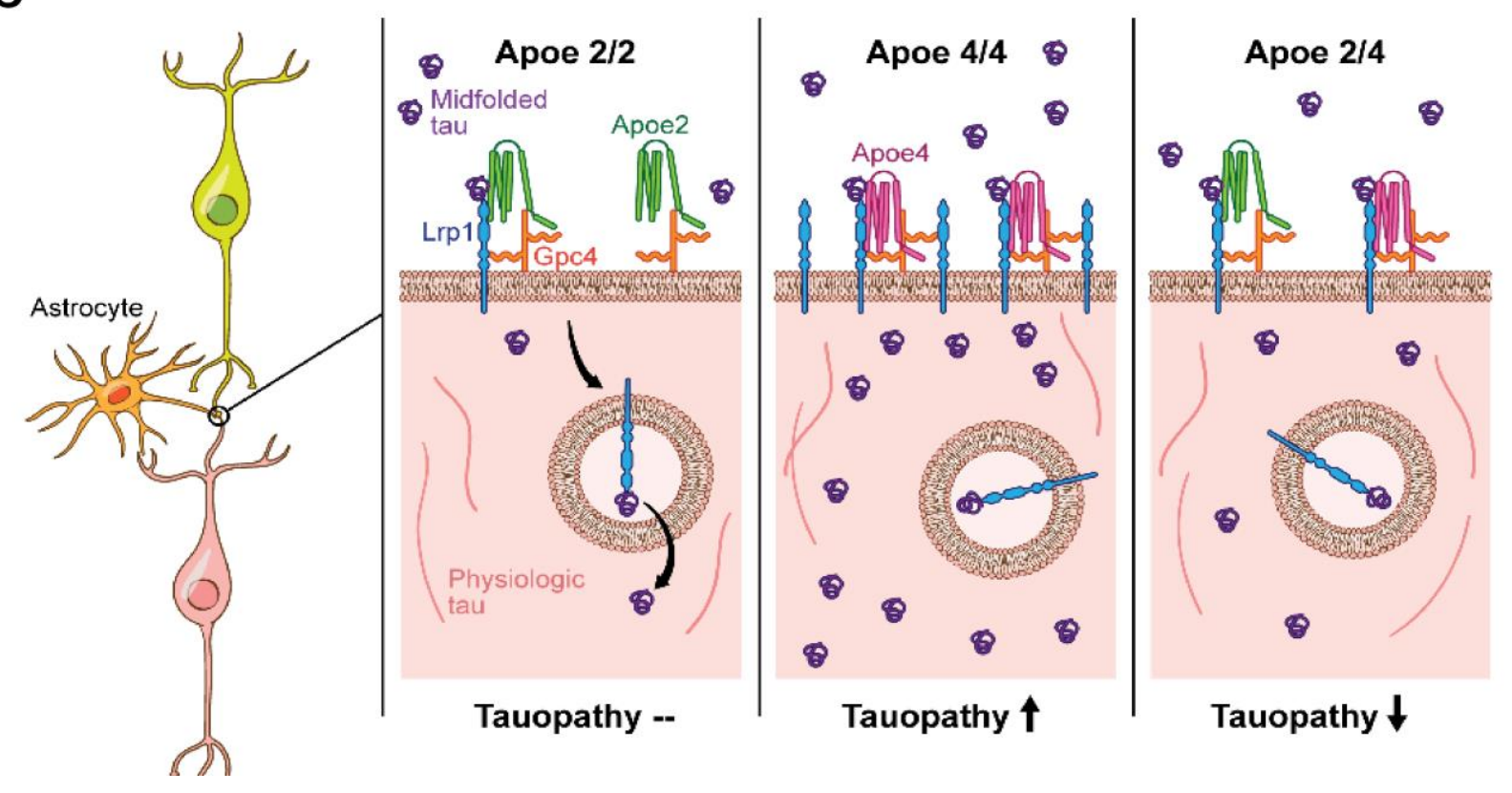

\section{Supplementary Figure 4}

A) Immunoprecipitation of human brain protein samples with APOE antibody and subsequent western blotting revealed that APOE and GPC-4 are in the same complex. B) GPC-4 was incubated either with APOE2 or APOE4 and separated by a native gel. Western blot analysis with GPC-4 and APOE antibodies show that GPC-4 strongly interacts with APOE4. 
A
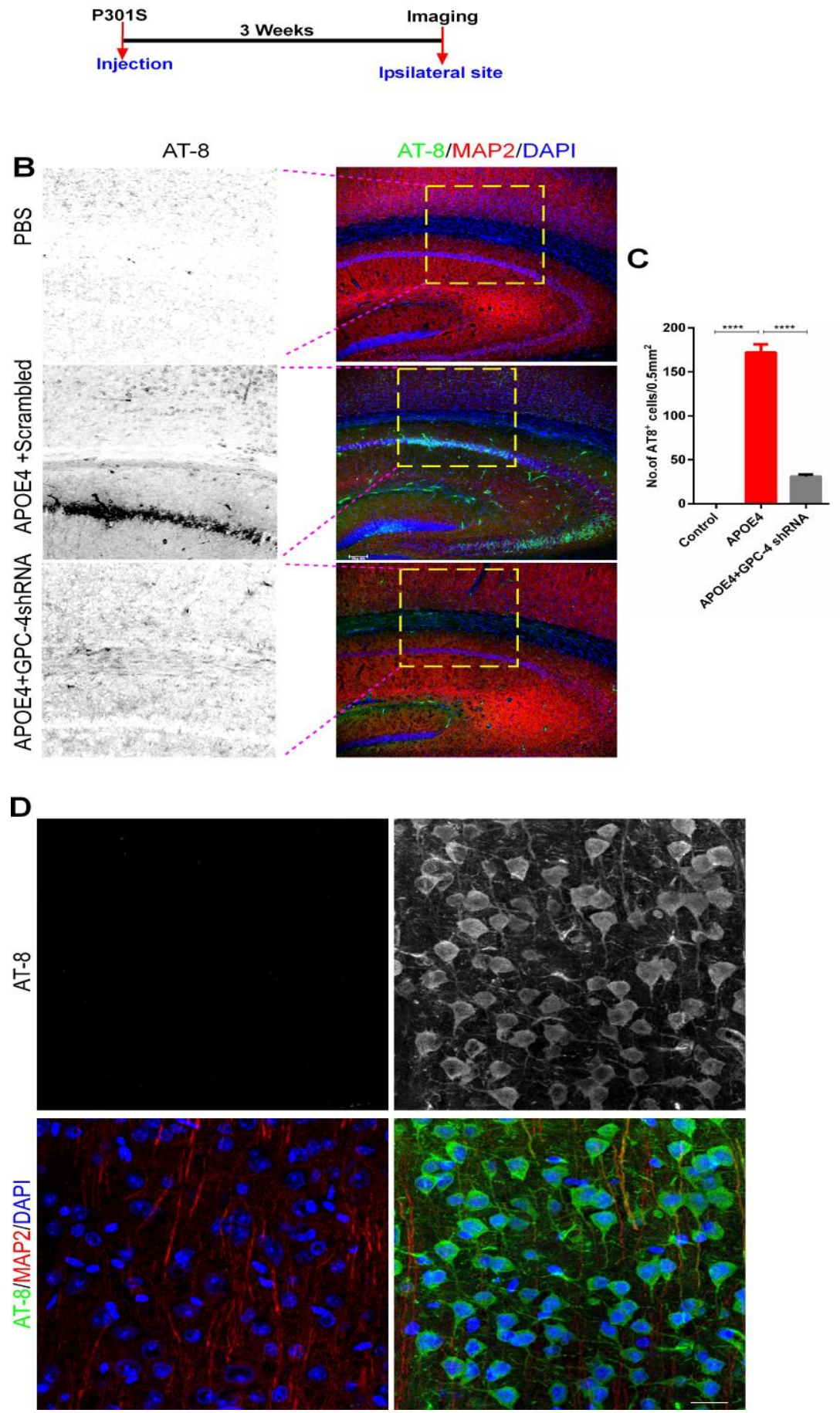

\section{Supplementary Figure 5}

A) A unilateral injection of APOE4 or GPC-4 shRNA in the CA1 region of P301S animals. B, C) APOE4 protein induced tau accumulation on ipsilateral hippocampal site, and the presence of GPC-4 shRNA halted APOE4 mediated tau accumulation. D) Representative IHC staining with AT8 antibody shows that GPC-4 induces tau accumulation in the cortical regions. 


\section{Materials and Methods}

\section{Astrocyte culture and preparation of ACM}

The procedures were adapted from previous publication ${ }^{33}$. Cortical astrocytes were cultured in DMEM supplemented with 10\% FBS, Glutamax, sodium pyruvate, insulin and Penicillin/Streptomycin. Once it reached the confluences, cells were washed with PBS, added astrocyte-conditioned medium (ACM), and incubated for three days. For shRNA experiments, either GPC-4 shRNA or scrambled shRNA was added to astrocyte culture when incubated with ACM to knock-down GPC-4. At the end of day 3, the medium was collected, concentrated/dialyzed using $10 \mathrm{kD}$ Amicon ${ }^{\circledR}$ Ultra Centrifugal filter tubes. The concentrated ACM was added to neuronal culture.

\section{Neuronal culture}

Cortical neuronal culture was performed from wild-type or P301S or Tau KO P1 pups as indicated throughout the manuscript. The procedures were performed as described previously with modifications ${ }^{50}$. Following dissection in Hybernate A solution, cortex was digested for 30 min in papain. Papain solution was inactivated with ovomucoid inhibitor, tissues were dissociated, and passed through a $20-\mu \mathrm{m}$ mesh filter. To remove the cell debris and immune cells, we performed negative immunopanning in Lectin 1 coated dishes with goat-anti mouse $\operatorname{IgG}+\operatorname{IgM}$ and goat-anti rat IgG antibodies. A positive immunopanning with neural cell adhesion molecule L1 antibody was performed to enrich the neurons. Neurons were cultured in Neurobasal ${ }^{\mathrm{TM}}$ Plus Medium supplemented with B27, Glutamax and Penicillin/Streptomycin. A half-of medium was replaced every 3 days. To study an effect of the APOE variants or GPC-4 on tau pathology, neurons were treated on day 11 with $5 \mu \mathrm{g} / \mathrm{ml}$ of protein for 4 days. To investigate the role of APOE variants or GPC-4 on surface LRP1, neurons were treated for $24 \mathrm{~h}$. GPC-4 protein was purified from frozen human tissues. The APOE proteins were purchased from Sigma, Abcam and R\&D systems. Agrin, Syndecan-3 and Glypican-3 proteins were purchased from R\&D systems. To study the role of ACM on tau pathology, neurons were treated with ACM for 4 days.

\section{Isolation of surface proteins}

To isolate cell surface proteins, Neurons were plated in 24-well plates. The cells from three wells/condition were pooled together. Cell surface proteins were biotinylated and isolated using 
Pierce Cell Surface Protein Isolation Method. All the steps were performed at $4^{0} \mathrm{C}$ unless otherwise mentioned. The cells were washed briefly with PBS and incubated with Sulfo-SS-Biotin for 30 mins. After the reaction was quenched, the cells were centrifuged at 1,200rpm for 4mins, and the resulting pellet was lysed with a lysis buffer and incubated for $1 \mathrm{~h}$. Some fraction of samples was collected at this stage to evaluate the total protein levels. After $1 \mathrm{~h}$ incubation with a lysis buffer, samples were centrifuged at $12,000 \mathrm{rpm}$ for 5 mins. The supernatant was incubated with NeutrAvidin Agarose for $1 \mathrm{~h}$ in a rotating shaker. Unbound proteins were washed with a wash buffer 3 times. The slurry containing proteins were boiled for $5 \mathrm{mins}$ at $95^{\circ} \mathrm{C}$ with $50 \mathrm{mM}$ DTT containing SDS sample buffer. Finally, it was centrifuged at 1,200 rpm for 2 mins to collect the proteins.

\section{Animals}

All animal procedures were performed according to the Icahn School of Medicine at Mount Sinai Institutional Animal Care and Use Committee (IACUC) guidelines. 4 months old P301s animals were used for the following experiments. For GPC-4 injection, $4 \mu \mathrm{g}$ of GPC-4 protein was injected in the right CA1 region (AP-2, ML-1.3 and DV-1.5). And the volume of PBS was injected on the left CA1 hippocampal regions. After one week of incubation period, animals were sacrificed and processed for IHC. In another study, 4ug of APOE4 was unilaterally injected in CA1 regions. After $24 \mathrm{~h}$, one group (control) received scrambled scRNA alone, one group of APOE4 injected animals received scrambled scRNA and another APOE4 injected group received GPC-4 shRNA. Animals were sacrificed after 3 weeks of injection for further analysis.

\section{Protein binding assays}

To investigate whether GPC-4 differentially interacts with APOE2 and APOE4, the protein binding assay was performed. The concentration of any indicated protein was at $1 \mu \mathrm{g} / \mu \mathrm{l}$. The final volume was made to five microliters with Tris buffer. The mixture was incubated at a shaker for one hour. The samples were boiled with or without 2-mercaptoethanol and separated by a native gel. 


\section{Immunoprecipitation}

The total protein was isolated from frozen human brain samples using 1\% Triton in Tris buffer. Briefly, Pierce Direct IP method was used to isolate proteins of interest. The AminoLink Plus Coupling Resin was washed with a coupling buffer, and the resin was incubated with $10 \mu \mathrm{g}$ of LRP1 or APOE antibodies for $2 \mathrm{~h}$ in a rotator. The unbound antibodies were washed away, and $500 \mu \mathrm{g}$ of isolated proteins was added to resin and incubated overnight, $4^{0} \mathrm{C}$, in a rotator. It was washed three times to remove unbound proteins, and antibody-coupled proteins were eluted with an elution buffer.

\section{Ion exchange chromatography}

The method was adapted from previous publication ${ }^{14}$. The DEAE Sepharose was equilibrated with a wash buffer. Ten micrograms of GPC-4 protein were added to the column and incubated for $5 \mathrm{~h}$ at $4{ }^{0} \mathrm{C}$. After incubation, the unbound GPC-4 was washed away, 5 times, with a washing buffer. And then, either APOE2 or APOE4 protein was added and incubated for $1 \mathrm{~h}$. After washing, 5 times, the bound proteins were eluted with $\mathrm{NaCl}$ gradients. The excess salt was removed by using $10 \mathrm{kD}$ Amicon ${ }^{\circledR}$ Ultra Centrifugal filter tubes.

\section{Electrophoresis and western blot}

Samples were lysed with SDS-lysis buffer (Laemmli Sample Buffer, Biorad), boiled at $95^{\circ} \mathrm{C}$ for 5 mins with $50 \mathrm{mM}$ DTT, and separated based on their molecular weights using TGX Stain-Free Gels (Biorad). The separated proteins were transferred to PVDF membrane, blocked with 2\% BSA for $1 \mathrm{~h}$, incubated with primary antibodies overnight at $4^{0} \mathrm{C}$. Membranes were washed with PBST 6 x10 mins, incubated with HRP-conjugated secondary antibodies for $1 \mathrm{~h}$, again washed with PBST 6 x10 mins and then developed using ECL solution (Clarity Western ECL substrate, Biorad). The following primary antibodies were used: TAU-5 (Thermo Scientific), AT-8 (Thermo

Scientific), Actin (Cell Signaling), LRP1 (Abcam), APOE (Abcam), Glypican-4 (Thermo Scientific and Sigma), Agrin (Abcam), Syndecan-3 (Abcam) and Glypican-3 (Abcam).

\section{Tau uptake assay}

On day 11 of neuronal culture, the cells were treated either with GPC-4 shRNA, LRP1 shRNA or scrambled shRNA. After $24 \mathrm{~h}$ of treatment, it was incubated with $5 \mu \mathrm{g} / \mathrm{ml}$ APOE4 protein for 24 
$\mathrm{h}$ as described in the manuscript. Finally, the neurons were incubated with $1 \mu \mathrm{g} / \mathrm{ml}$ of human tau protein for $1 \mathrm{~h}$ and washed with PBS 3 times before proceeding to immunohistochemistry.

\section{Tau seeding assay}

The Tau Biosensor (ATCC CRL-3275) cells stably expressing the repeat domain of Tau conjugated yellow fluorescent protein (YFP) were cultured in 24 well-coverslips at $37{ }^{\circ} \mathrm{C}, 5 \% \mathrm{CO} 2$ in a complete medium. Brain extracts from Alzheimer's disease patients were used. APOE4contaning particles were isolated from APOE44 postmortem human brain. 3\% (final volume) Lipofectamine 2000 were mixed with brain extracts alone, brain extracts + isolated APOE4 particles and brain extracts + purified APOE4 protein for one hour at room temperature. It was added to the CRL cells, incubated for two days, fixed with 4\%PFA for one hour and YFP signals were imaged at SP5 Leica microscope. The tau seeded cells were blindly counted.

\section{Immunohistochemistry and imaging}

The cultured cells containing coverslips were fixed for an hour with $4 \%$ paraformaldehyde. After a brief wash with PBS, incubated with 10\% Donkey serum in PBST for 30 min. Primary antibodies were added and incubated for $48 \mathrm{~h}$ at $4^{0} \mathrm{C}$, washed $3 \times 10$ mins with PBST, probed with corresponding secondary antibodies for $2 \mathrm{~h}$, then washed again with PBST before counterstaining with DAPI. The tissues were mounted with Aqua-Poly/Mount and imaged using Leica TCS SP5 confocal laser scanning microscope. The following primary antibodies were used: TAU-5 (Thermo Scientific), AT8 (Thermo Scientific), MAP2 (Millipore), LRP1(Abcam), HT-7 (Thermo Scientific) and GFP (Abcam).

\section{Data and statistical analysis}

Quantification of western blots and immunocytochemical staining were performed by Image J software. For western blot quantification, the intensity of each immunoreactive band was normalized to the corresponding beta-actin immunoreactive band. Normalized values were grouped and comparted for statistical analysis. To analyze tau uptake (HT-7 staining), we imaged the neurons with 40x objective. Before staining, the coverslips were washed thoroughly with PBS to remove any extracellular tau. For different places/coverslip were imaged. The whole field of imaged area was quantified. .scRNAseq data was analyzed using a pipeline provided by the 
authors ${ }^{35}$. Statistical analyses were performed with Graphpad prism software. Unpaired student's t-test was used for two samples. For more than two samples, One-way ANOVA with Bonferroni correction was used. 
Figures

Figure 1
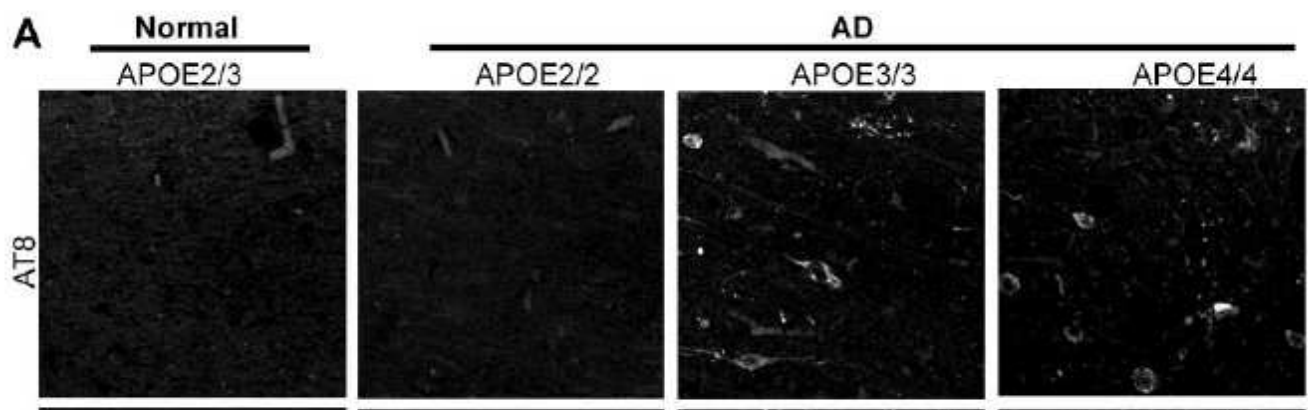

\section{B}
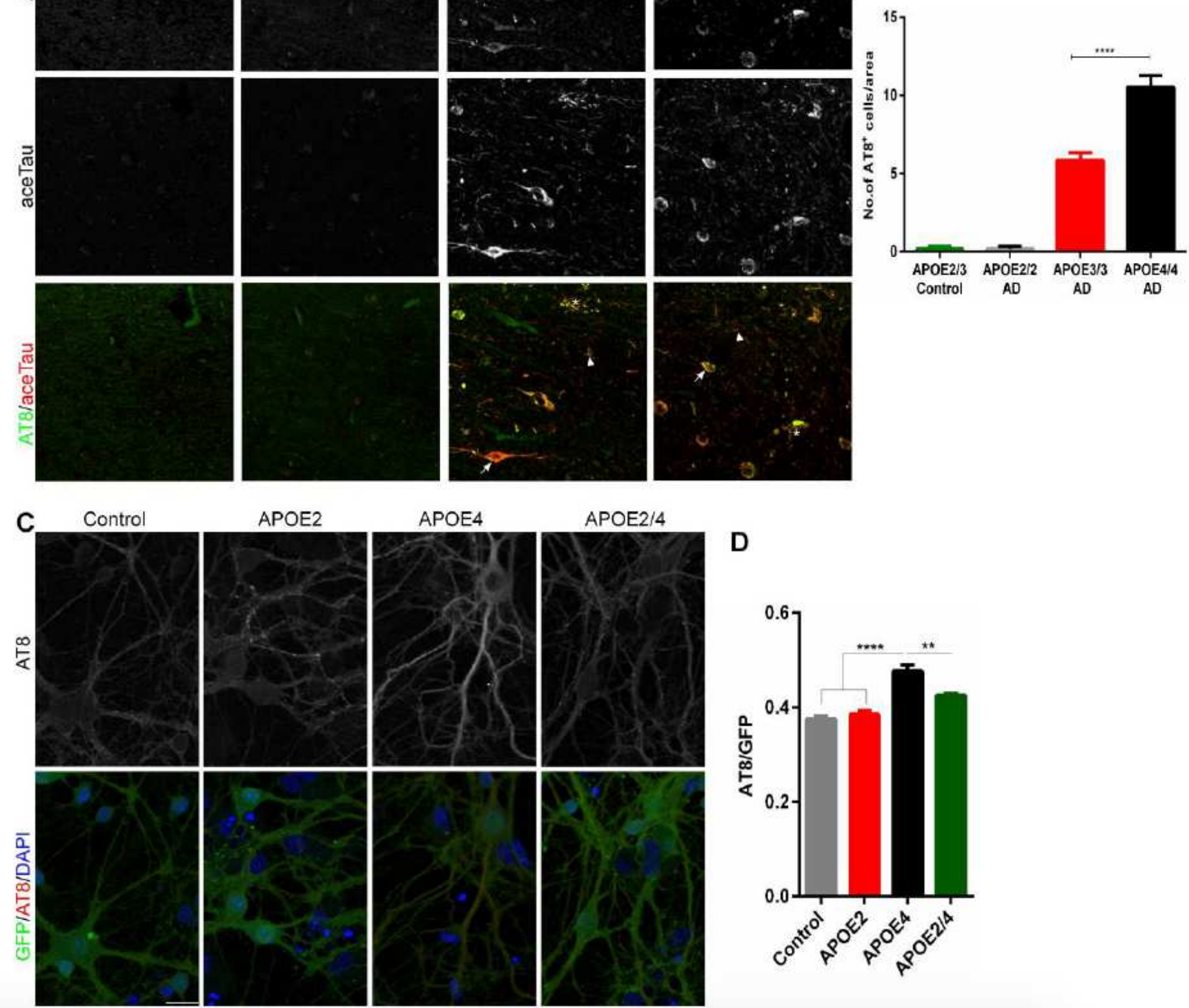

APOE2/4

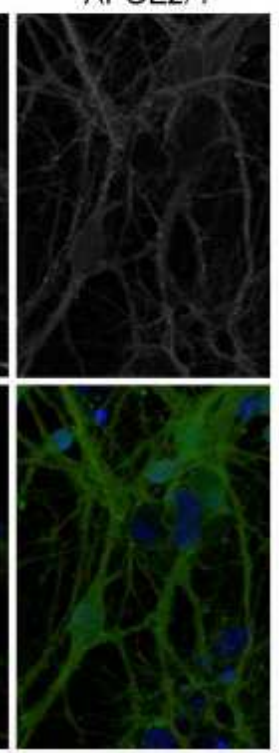

D

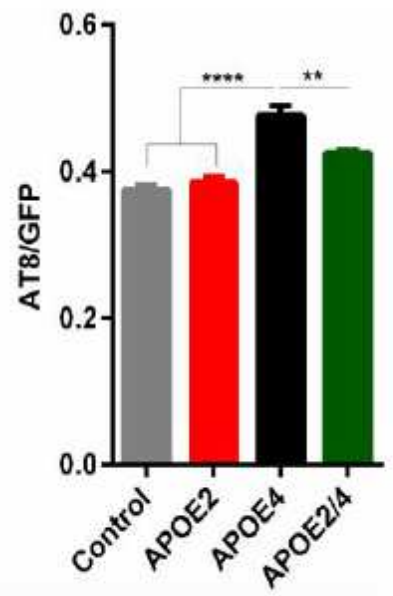

\section{Figure 1}

APOE4 AD patients have more tau accumulation and APOE4 enhance tau propagation. A) Representative IHC images of postmortem tissues from APOE2/3(control), APOE2/2(AD), APOE3/3 (AD) and APOE4/4 (AD) individuals stained with AT8 and aceTau (Lys174) antibodies ( $n=5-7)$. Note the presence of Neurofibrillary tangles (arrow), neuropil threads (asterisk) and neuritic plaque (arrowhead). B) Compared 
to APOE3/3 and APOE2/2, APOE4/4 shows presence of more neurofibrillary tangle-containing neurons. C) Representative IHC staining of APOE treated neuronal culture (Tau P301S* Tau KO animals) shows that APOE 4 treatments enhanced tau spreading whereas APOE2 significantly reduced APOE4-mediated tau spreading (D). $n=3-4$, one-way ANOVA, mean \pm SEM, IHC scale bars $=20 \mu \mathrm{m}$. ${ }^{\star \star} P<0.01,{ }^{\star \star \star *} P<0.001$ and $\star \star \star * P<0.0001$

\section{Figure 2}

A
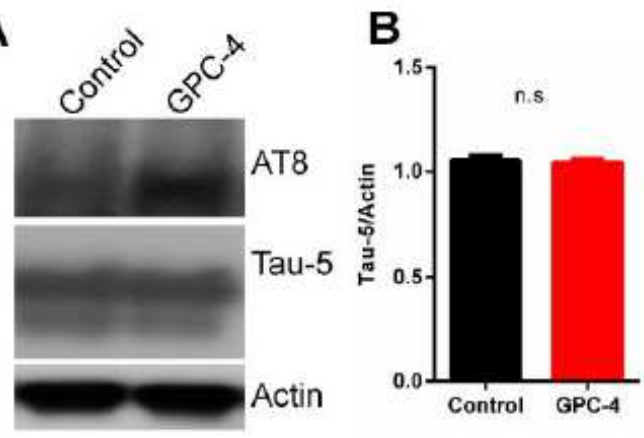

$\mathbf{F}$

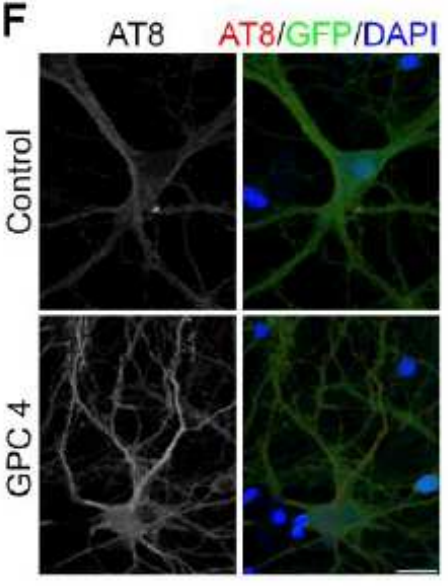

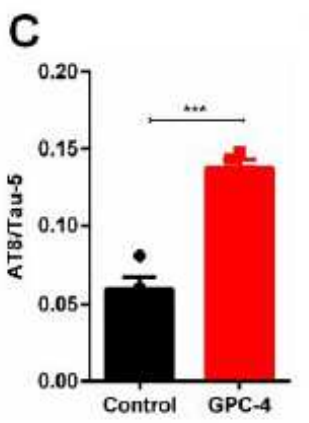

G

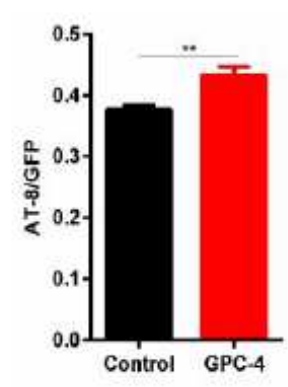

H
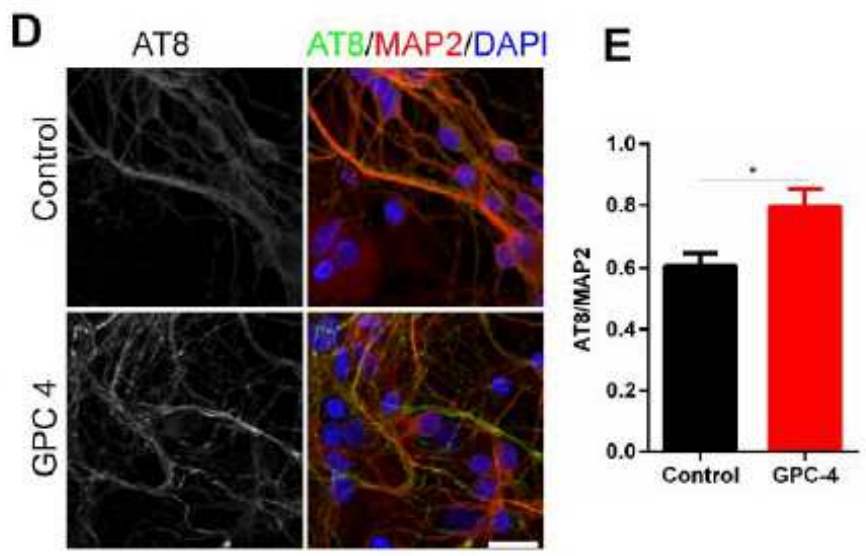

K

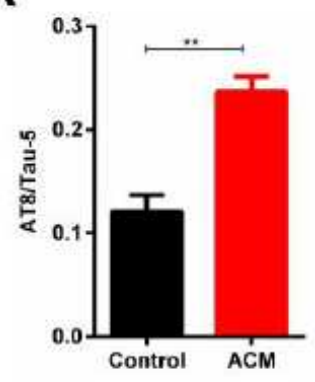

L

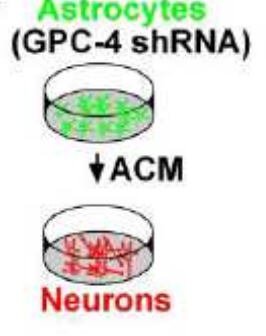

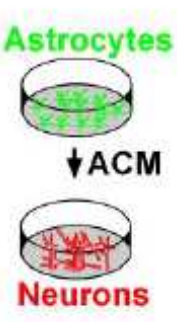

Astrocytes

I
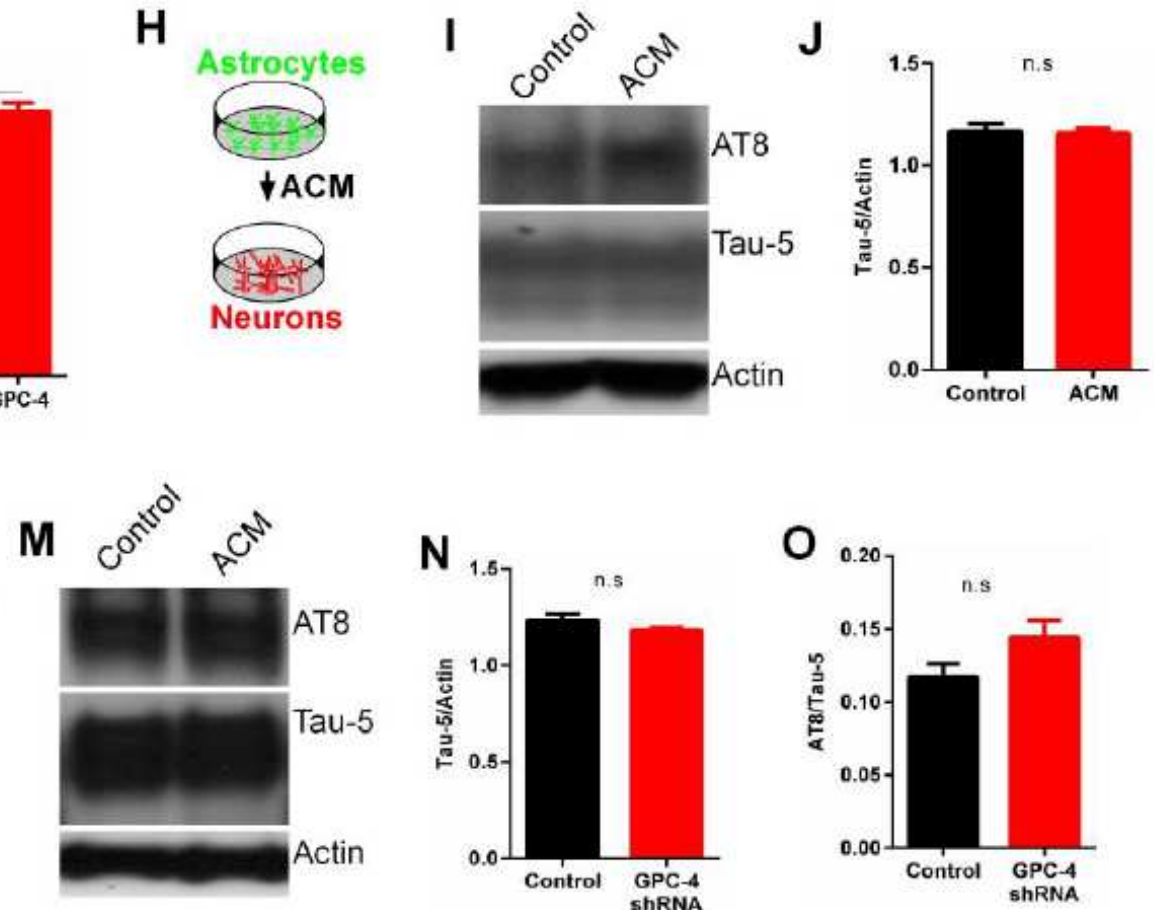
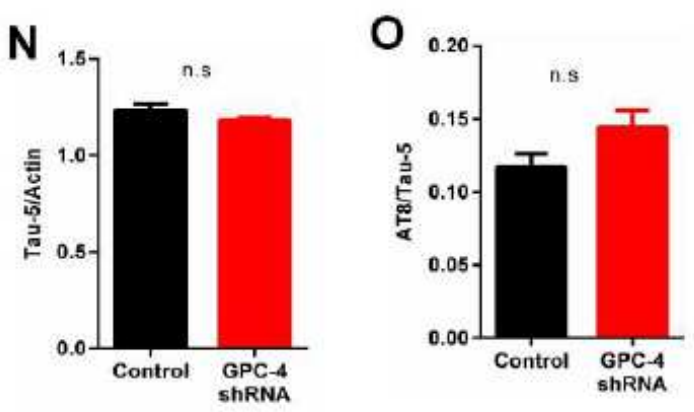

Figure 2

Astrocyte -secreted Glypican-4 drives tau pathology A) Western blot analysis of tau protein from neuronal culture (Tau P301S animals) treated with GPC-4 protein shows that GPC-4 significantly enhanced pTau levels (C) whereas no changes observed in total tau protein (B). D) Representative IHC staining of GPC-4 treated neuronal culture (Tau P301S animals) with AT8, MAP2 and DAPI demonstrates that GPC-4 
treatment enhanced AT8 level in neurons (E). F) Representative IHC staining of GPC-4 treated neuronal culture (Tau P301S* Tau KO animals) shows that GPC-4 treatment enhances tau spreading (G). H) Schematic diagram shows that astrocyte-conditioned media (ACM) from astrocyte culture was added to neuronal culture. I-K) Addition of ACM to neuronal culture increased AT8 levels whereas total tau was unaltered. L) Schematic diagram shows that astrocytes were treated with GPC-4 shRNA and the resulting GPC-4 deprived ACM was added to neuronal culture. M-0) Addition of GPC-4 deprived ACM failed to induce tau phosphorylation in neurons. $n=3-4$, unpaired Student's t-test, mean $\pm S E M$, IHC scale bars $=20$ $\mu \mathrm{m} .{ }^{*} \mathrm{P}<0.05,{ }^{*} \mathrm{P}<0.01$, and ${ }^{* * * P}<0.001$ 
Figure 3

A
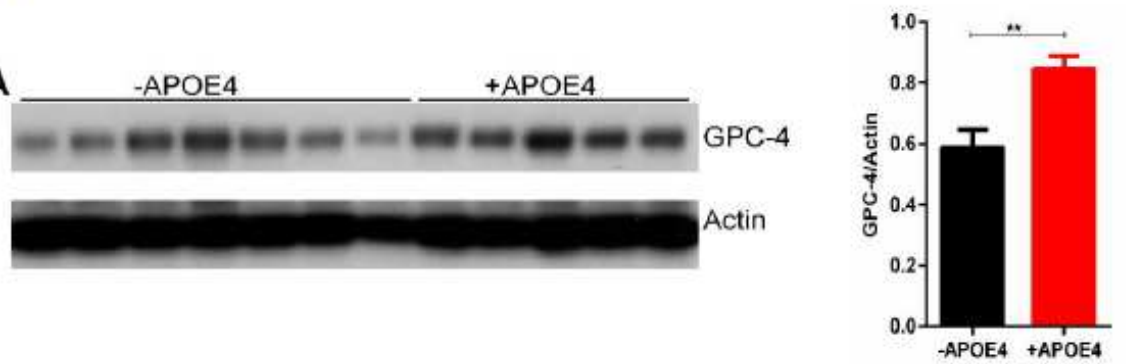

B
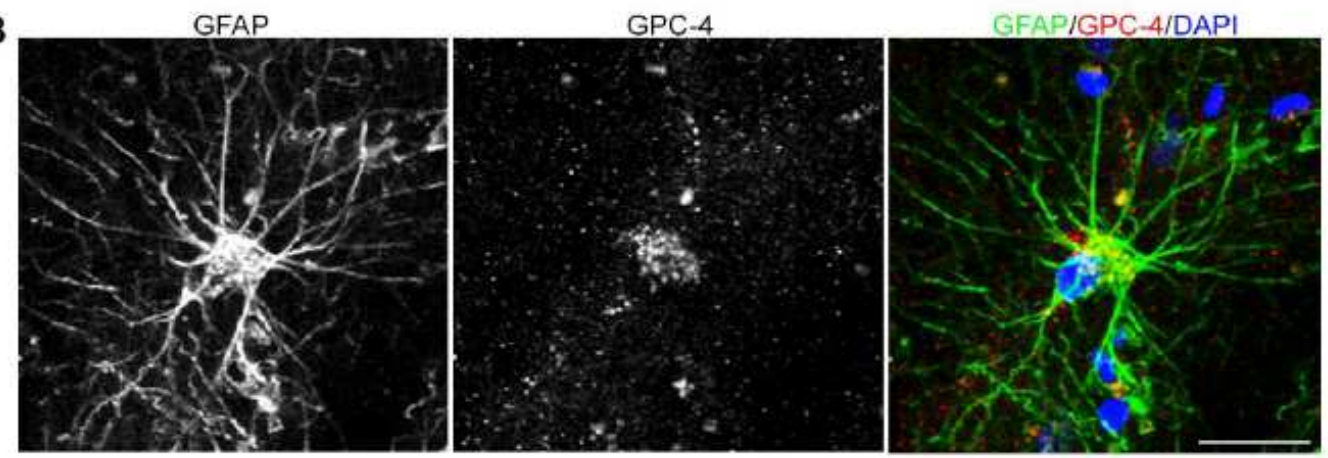

C
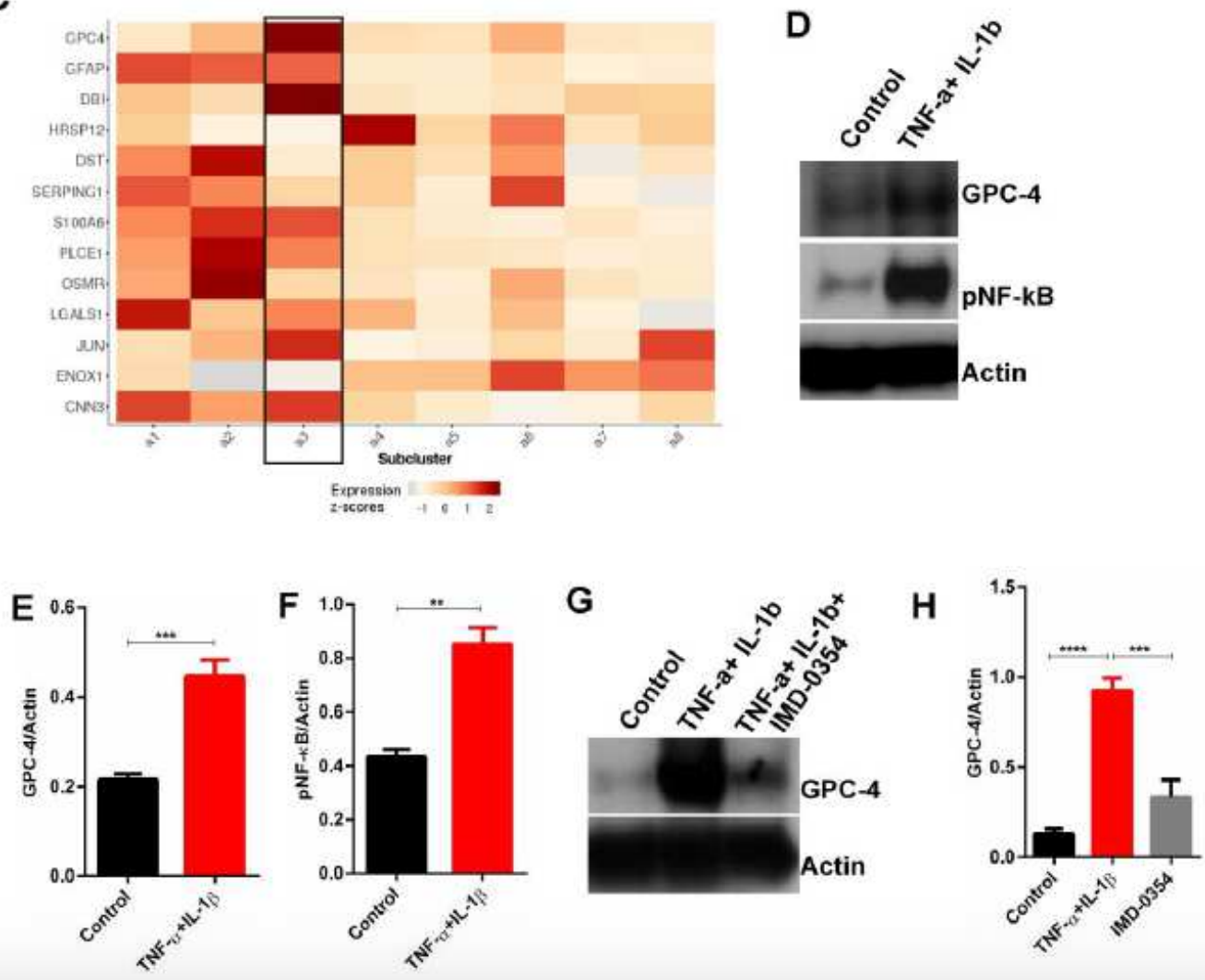

\section{Figure 3}

GPC-4 is expressed in a subtype of neurotoxic astrocytes. A) Western blot analysis from APOE44-carrying $A D$ patients and APOE4-noncarrying AD patients show that GPC-4 is up-regulated in APOE4 carrying individuals. B) Immunohistochemistry from the human brain shows that GPC-4 is expressed in GFAP astrocytes. C) Heat map shows that GPC-4 is enriched in AD-Associated astrocytes. D, E) Western blot analysis from astrocyte culture shows that treatment with TNF- $\alpha$ and IL-1 $\beta$ significantly increased 
expression of GPC-4 and activated NF-kB pathway. G, H) Western blot analysis from astrocyte culture treated NF-KB pathway blocker IMD-0354 shows that NF-kB pathway-dependent expression of GPC-4. $n=3-4$, unpaired Student's t-test, mean $\pm S E M,{ }^{* *} P<0.01,{ }^{* \star *} P<0.001$ and ${ }^{* \star \star *} P<0.0001$. IHC scale bars $=20$ $\mu \mathrm{m}$.

\section{Figure 4}
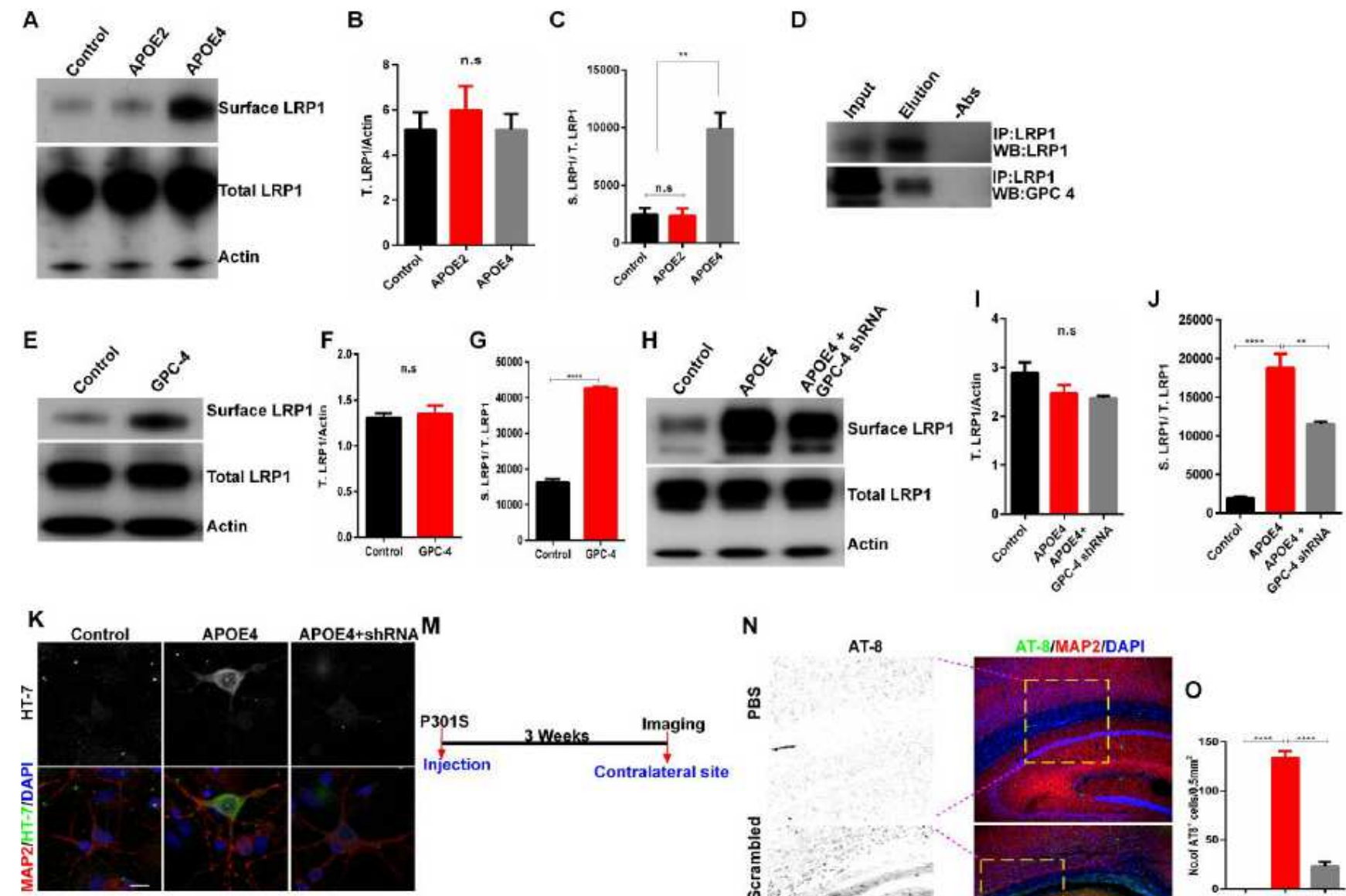

$\mathbf{L}$

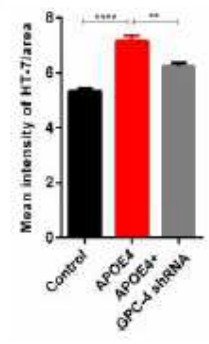

N
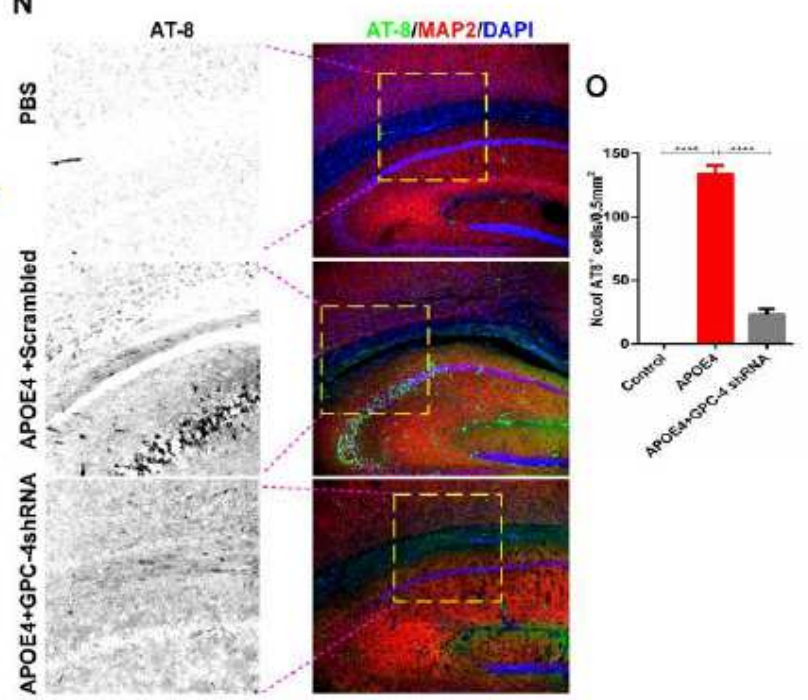

Figure 4

GPC-4 is required for APOE 4-mediated LRP1 trafficking and tau pathology A-C) Western blot analysis from primary neuronal culture treated with APOE proteins show that APOE4 significantly enhances surface LRP1 (S.LRP1) (B). There were no changes in total LRP1 (T.LRP1) (C). D) Immunoprecipitation of human postmortem brain protein samples with LRP1 antibody and subsequent western blotting revealed that LRP1 and GPC-4 are in the same complex. E-G) Western blot analysis from neuronal culture treated with GPC-4 protein shows that GPC-4 significantly enhanced trafficking of surface LRP1 levels (G), 
whereas total LRP1 levels were not affected (F). H-J) Western blot analysis from neuronal culture treated with APOE4 and with GPC-4 shRNA shows that APOE4 induced surface expression of LRP1 is mediated by GPC-4. The GPC-4 shRNA treatment reduced the surface expression of LRP1 in the presence of APOE4 compared to APOE4 alone $(\mathrm{J})$. K) Representative IHC staining of APOE4 treated neuronal culture shows that APOE4 treatment enhanced tau uptake and presence of GPC-4 shRNA did the converse, suggesting that GPC-4 is required for APOE4-mediated tau uptake (L). C) A unilateral injection of APOE4 or GPC-4 shRNA in the CA1 region of P301S animals. N, 0) APOE4 protein induced tau tau spreading to contralateral hippocampus. However, the presence of GPC-4 shRNA halted APOE4 mediated tau accumulation and spreading. $n=3-5$, one-way ANOVA, mean \pm SEM, IHC scale bars $=20 \mu \mathrm{m}$ (C) or $100 \mu \mathrm{m}$ (E). ${ }^{* *} \mathrm{P}<0.01$ and ${ }^{* \star * *} \mathrm{P}<0.0001$. 


\section{Figure 5}

A

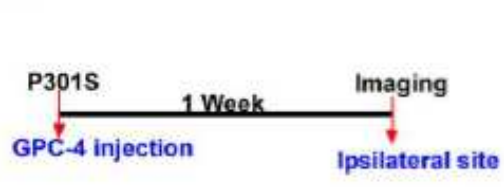

B

Control

GPC-4
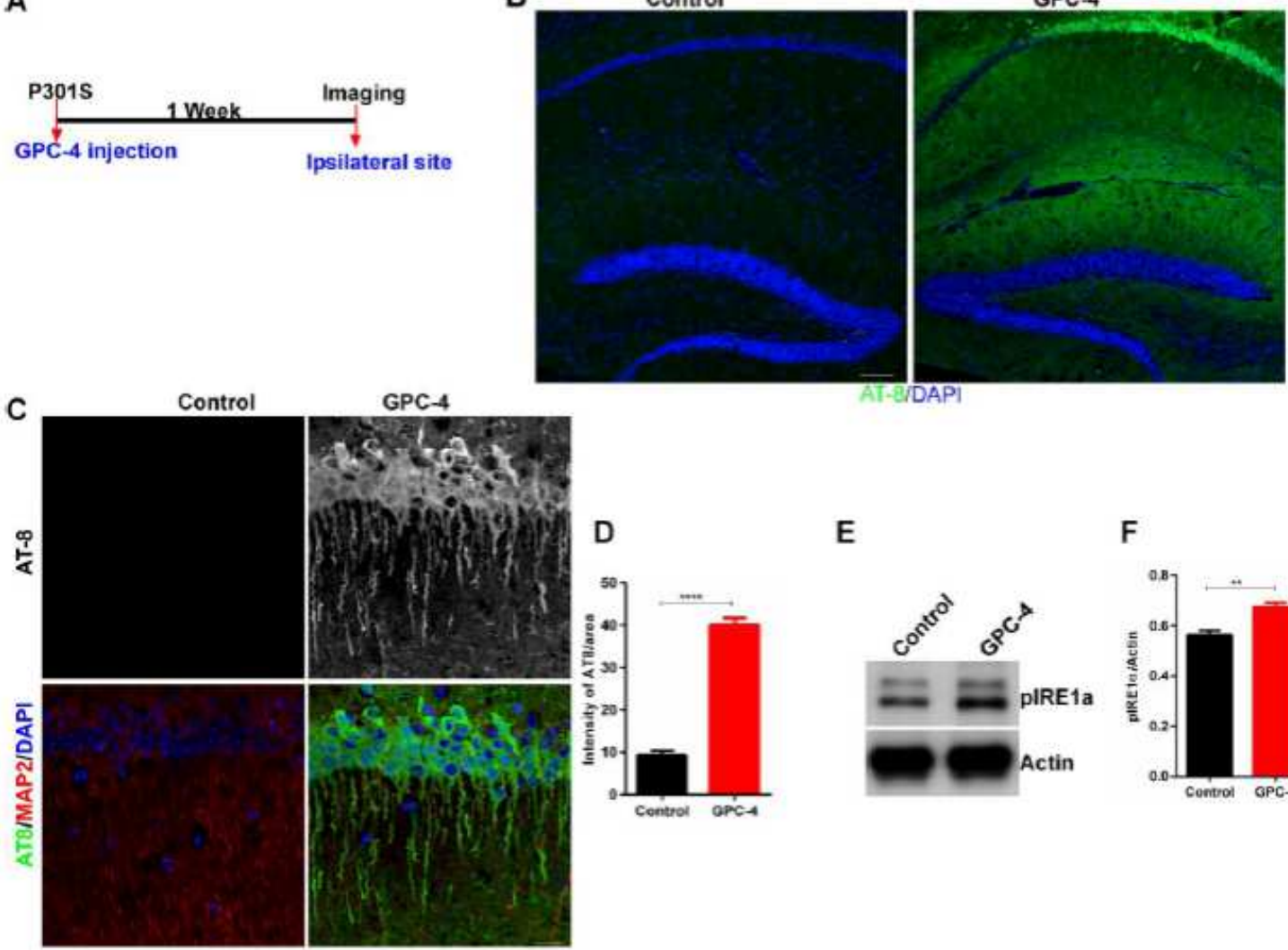

D E

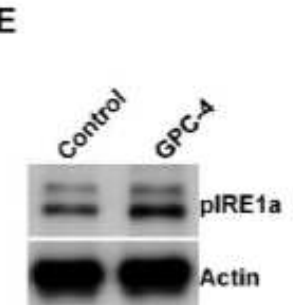

F
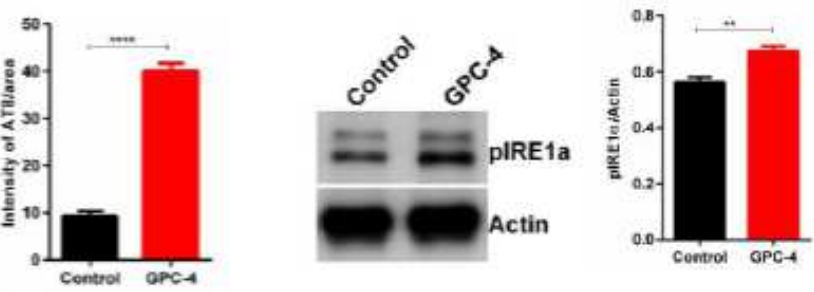

H

AT8

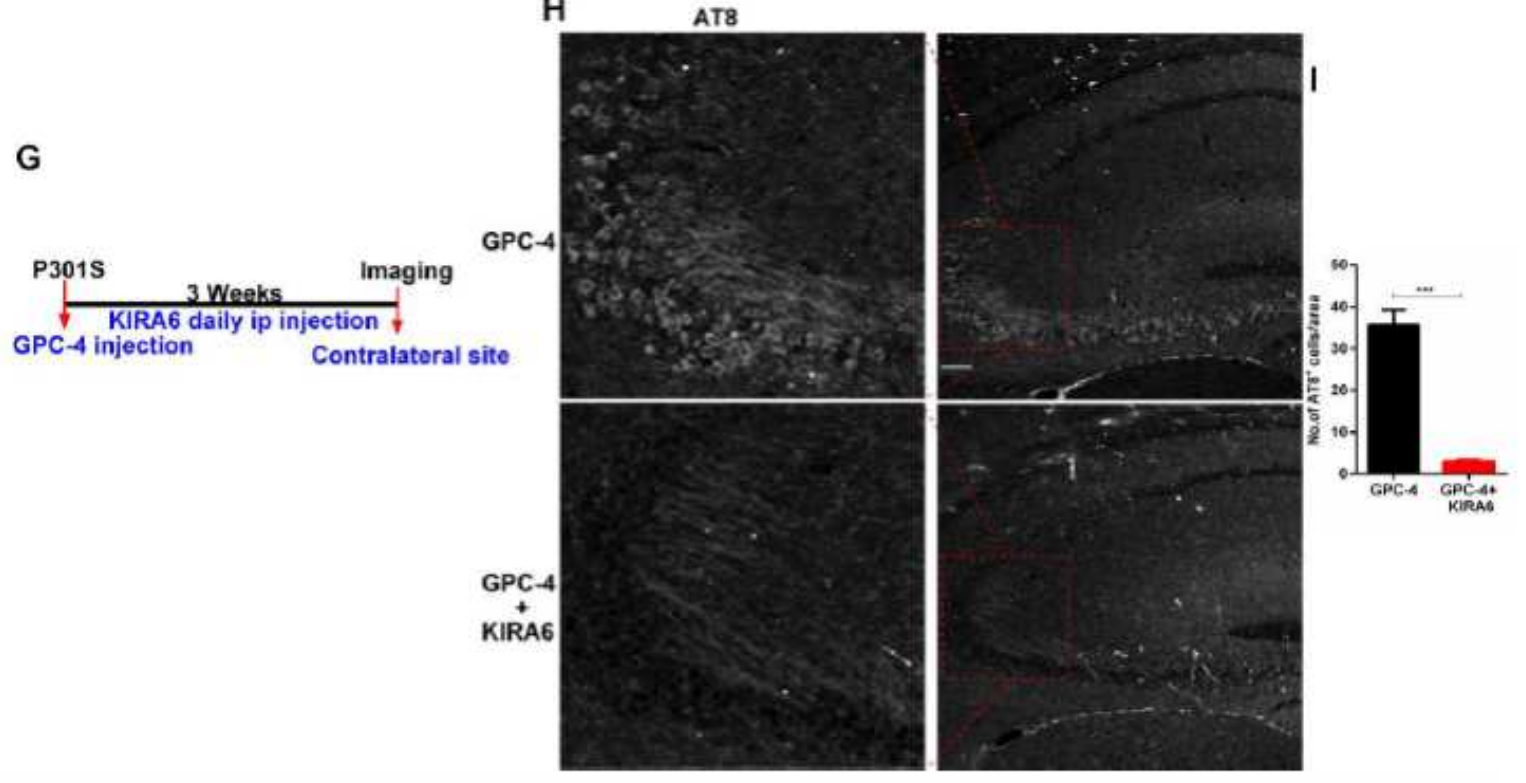

\section{Figure 5}

Inhibition of IRE1 a pathway blocks GPC-4 mediated tau propagation. A) A unilateral injection of GPC-4 protein in the CA1 region of P301S animals and analysis after a week. B-D) After one week of incubation period, elevated levels of tau accumulation were observed in GPC-4 injected hippocampal regions. E) Cultured primary neurons were treated with GPC-4 for $1 \mathrm{hr}$ and performed a western blotting. GPC-4 treatment shows a significant activation of IRE1 a pathway (F). G) A unilateral injection of GPC-4 protein 
in the CA1 region of P301S animals and daily ip injection IRE1 a pathway inhibitor KIRA6 for 3 weeks. $\mathrm{H}, \mathrm{I}$ ) After 3 weeks, GPC-4 induced tau propagation on contralateral sites and KIRA6 treatment blocked GPC-4 induced tau propagation. . $n=3-5$, unpaired Student's t-test, mean $\pm S E M, * \star P<0.01$, ${ }^{\star \star \star} P<0.001$ and $\star \star \star \star P<0.0001$. IHC scale bars $=20 \mu \mathrm{m}$ and $100 \mu \mathrm{m}$. 